\title{
Primordial hypermagnetic fields and the triangle anomaly
}

\author{
M. Giovannini* \\ DAMTP, Silver Street, CB3 9EW Cambridge, United Kingdom \\ M. E. Shaposhnikov ${ }^{\dagger}$ \\ CERN, Theory Division, CH-1211 Geneva 23, Switzerland
}

(Received 3 October 1997; published 2 February 1998)

\begin{abstract}
The high-temperature plasma above the electroweak scale $\sim 100 \mathrm{GeV}$ may have contained a primordial hypercharge magnetic field whose anomalous coupling to the fermions induces a transformation of the hypermagnetic energy density into fermionic number. In order to describe this process, we generalize the ordinary magnetohydrodynamical equations to the anomalous case. We show that a not completely homogeneous hypermagnetic background induces fermion-number fluctuations, which can be expressed in terms of a generic hypermagnetic field configuration. We argue that, depending upon the various particle physics parameters involved in our estimate (electron Yukawa coupling, strength of the electroweak phase transition) and upon the hypermagnetic energy spectrum, sizable matter-antimatter fluctuations can be generated in the plasma. These fluctuations may modify the predictions of the standard big bang nucleosynthesis (BBN). We derive constraints on the magnetic fields from the requirement that the homogeneous BBN is not changed. We analyze the influence of primordial magnetic fields on the electroweak phase transition and show that some specific configurations of the magnetic field may be converted into net baryon number at the electroweak scale. [S0556-2821(98)05704-X]
\end{abstract}

PACS number(s): 98.80.Cq, 98.80.Ft

\section{INTRODUCTION}

There are no compelling reasons why magnetic fields should not have been present in the early universe. Moreover, it can be argued that the existence of some magnetic fields at high temperatures is a quite natural phenomenon. Indeed, the presence of large-scale magnetic fields in our observed universe is a well established experimental fact. Since their first evidence in diffuse astrophysical plasmas beyond the solar corona [1,2], magnetic fields have been detected in our galaxy and in our local group through Zeeman splitting and through Faraday rotation measurements of linearly polarized radio waves. The Milky Way possesses a magnetic field whose strength is of the order of the microgauss corresponding to an energy density roughly comparable with the energy density today stored in the cosmic microwave background radiation (CMBR) energy spectrum peaked around a frequency of $30 \mathrm{GHz}$. Faraday rotation measurements of radio waves from extragalactic sources also suggest that various spiral galaxies are endowed with magnetic fields whose intensities are of the same order as that of the Milky Way [3]. The existence of magnetic fields at even larger scales (intergalactic scale, present horizon scale, etc.) cannot be excluded, but it is still quite debatable since, in principle, dispersion measurements (which estimate the electron density along the line of sight) cannot be applied in the intergalactic medium because of the absence of pulsar signals [3].

If the existing galactic magnetic field is naively blueshifted to earlier epochs, one then finds that the universe was

\footnotetext{
*Electronic address: m.giovannini@damtp.cam.ac.uk

${ }^{\dagger}$ Electronic address: mshaposh@nxth04.cern.ch
}

always filled by a magnetic field of a considerable amplitude, with energy density of the order of the energy density of $\gamma$ quanta. Of course, this consideration does not take into account different physical processes operating at the galactic scale, such as the dynamo mechanism $[2,4]$ or the anisotropic collapse mechanism [5], which change considerably the naive scaling law $|\vec{H}| \sim T^{2}$. In any case, it is widely believed that some seed fields of primordial origin are necessary for the successful generation of the galactic magnetic fields [6].

Looking at this problem from a more theoretical side, there are several mechanisms that may successfully generate large enough magnetic seeds coherent on different scales. Magnetic seeds can be produced either during a first-order quark-hadron phase transition $[7,8]$ or during the electroweak phase transition [9-12]. Recently it was also suggested that a primordial asymmetry encoded in the rightelectron number can be converted in a quite large hypercharge seed during the symmetric phase of the electroweak theory [13]. The seeds could also be the result of the parametric amplification of the quantum mechanical (vacuum) fluctuations of some primordial gauge field, in the same way as in general relativity the quantum mechanical fluctuations of the tensor modes of the metric can be amplified, producing, ultimately, a stochastic gravity-wave background [14]. The essential ingredient of the large-scale magnetic field generation is the breaking of conformal invariance in the coupling of the electromagnetic field to gravity $[15,16]$. Reasonable seeds could also be produced if the inflaton is coupled to the Maxwell term in a chaotic inflationary scenario [17]. In the string theory low-energy effective action, the dilaton field provides a unified value of the gravitational and gauge coupling at the string scale and it naturally breaks the scale invariance of the electromagnetic and gauge couplings (also in four dimensions) without providing 
a gravitational mass for the photon. Sizable seeds, coherent over the galactic scale, can be generated $[18,19]$.

The possible existence of magnetic fields in the early universe has a number of interesting cosmological implications. For example, magnetic fields at small scales may influence the big bang nucleosynthesis (BBN) and change the primordial abundances of the light elements [20] by changing the rate of the universe expansion at the corresponding time. The success of the standard BBN scenario can provide an interesting set of bounds on the intensity of the magnetic fields at that epoch [20].

Long-range stochastic magnetic fields that possibly existed at the decoupling epoch might have induced anisotropies in the microwave sky [19]. The existence of a completely homogeneous field coherent over the horizon at the present epoch can be interestingly constrained by the Cosmic Background Explorer (COBE) observations [21,22]. Conversely, if the CMBR is linearly polarized, its polarization plane could have been rotated by the presence of a sufficiently energetic magnetic field coherent over the horizon size at the decoupling epoch [23]. Faraday rotation measurements applied to the galactic (synchrotron) emission can also provide interesting constraints [24] on large-scale magnetic fields (even though these are coherent over scales smaller than the present horizon).

In this paper we address the question of whether there can be any cosmological consequences from the fact that magnetic fields existed prior to the electroweak phase transition, when the background temperature was $T>T_{c} \sim 100 \mathrm{GeV}$. At these temperatures the electroweak symmetry is restored and the magnetic field is replaced by the hypermagnetic one. The hypercharge field, unlike the ordinary magnetic field, has an anomalous coupling to the fermions. This fact will play a crucial role in our considerations. The origin of primordial hypermagnetic fields is not essential for us and consequently we simply assume that they were generated by some mechanism before the electroweak (EW) phase transition.

We will show that, depending on the particle physics model and on the initial spectrum of the primordial magnetic fields, quite large fluctuations of the baryon and lepton numbers may be generated. These fluctuations can survive until the onset of BBN and create unusual initial conditions for the calculation of the light element abundances. In particular, a natural outcome of our considerations is a nonuniform distribution of baryon number, not necessarily positive definite. Matter-antimatter domains are then possible. The requirement that these fluctuations are small enough in order not to conflict with the predictions of the standard homogeneous BBN allows us to put quite a strong constraint on the spectrum of the primordial magnetic fields. Moreover, we will argue that the primordial magnetic field may change the nature of the electroweak phase transition. Finally, the existence of primordial fields with some specific topological structure may result in the production of the net baryon number at the electroweak scale.

The plan of the paper is the following. In Sec. II we derive our basic equations. For an ordinary electromagnetic plasma, it is fairly well established that the evolution of the magnetic fields can be described using the magnetohydrodynamical (MHD) equations [2,4,25]. In the case of a hightemperature electroweak plasma the MHD equations have to be generalized by taking into account anomaly effects. In the new AMHD equations (anomalous MHD equations) the magnetic hypercharge fields turn out to be coupled to the fermionic number density. As a consequence, the evolution equations of the anomalous charge densities acquire a magnetic source term. In Sec. III we describe an approximate solution of AMHD equations. We show that anomalous coupling gives rise to instabilities, allowing the conversion of the energy sitting in the fermionic degrees of freedom into magnetic hypercharge fields and vice versa. This phenomenon is completely absent in ordinary MHD. The presence of the fermionic number density produces a kind of "Ohmic" current, which is parallel to the magnetic hypercharge field. Also this phenomenon is quite new if compared with the ordinary MHD equations (though something vaguely similar can happen in the context of the dynamo mechanism [2] in a parity-noninvariant turbulent fluid). In Sec. IV we will apply our results to the case of stochastic hypercharge field backgrounds, whereas in Sec. V we focus our attention on the possible phenomenological implications of our considerations for BBN. Different bounds on primordial magnetic fields will also be analyzed in the light of our results. We will also discuss the dependence of the magnitude of the baryon-number fluctuations upon various particle physics parameters, which can appear in the extensions of the minimal standard model (MSM). In Sec. VI we discuss the influence of the primordial hypermagnetic fields on the dynamics of the electroweak phase transition, and we will show how some specific configurations of the hypermagnetic fields may create a net nonzero amount of baryons. Section VII contains our concluding remarks.

Some of the results of this paper have already been presented (in a more compact form) in [26] (see also the closely related paper [13] where transformation of a finite number density of right electrons into hypermagnetic fields has been considered).

\section{BASIC EQUATIONS}

\section{A. Preliminaries}

Let us start from some qualitative considerations. A unique property of "unbroken" $\mathrm{U}(1)$ gauge interaction is the absence of mass of its corresponding vector particle. Static "magnetic" fields are never screened (in the absence of monopoles) and thus homogeneous fields can survive in the plasma for infinitely long times. On the contrary, electric fields quickly decay because of the finite plasma conductivity $\sigma$ within a time scale $\sim 1 / \sigma$. Then long-ranged nonAbelian magnetic fields [corresponding to, e.g., the color $\mathrm{SU}(3)$ or weak SU(2) groups] cannot exist because at high temperatures the non-Abelian interactions induce a "magnetic" mass gap $\sim g^{2} T$. Also the non-Abelian electric fields decay because of the finite value of the conductivity as it occurs for Abelian electric fields. Therefore the only long scale field that can exist in the plasma for enough time must be associated with some Abelian U(1) group. This statement, valid to all orders in perturbation theory, has been confirmed nonperturbatively for the electroweak theory by recent lattice studies in [27]. Under normal conditions (i.e., small temperatures and small densities of the different fermionic charges) the $\mathrm{SU}(2) \times \mathrm{U}(1)_{Y}$ symmetry is "broken" down to $\mathrm{U}(1)_{\mathrm{em}}$, 
the massless field corresponding to $\mathrm{U}(1)_{\mathrm{em}}$ is the ordinary photon, and the only long-lived field in the plasma is the ordinary magnetic one. At sufficiently high temperatures, $T$ $>T_{c}$, the $\mathrm{SU}(2) \times \mathrm{U}(1)_{Y}$ symmetry is "restored," and nonscreened vector modes $Y_{\mu}$ correspond to the $\mathrm{U}(1)_{Y}$ hypercharge group. Hence, if primordial fields existed at $T>T_{c}$, they did correspond to hypercharge rather than to $\mathrm{U}(1)_{\mathrm{em}}$.

There are essential differences between the interactions of magnetic fields and those of hypermagnetic fields with matter. The ordinary electromagnetic field has a vectorlike coupling to the fermions, while the coupling of the hypercharge fields is chiral. Thus, if hyperelectric $\left(\overrightarrow{\mathcal{E}}_{Y}\right)$ and hypermagnetic $\left(\overrightarrow{\mathcal{H}}_{Y}\right)$ fields are present simultaneously, they cause a variation of the fermionic number according to the anomaly equation, $\partial_{\mu} j_{\mu} \sim\left(g^{\prime 2} / 4 \pi^{2}\right) \overrightarrow{\mathcal{H}}_{Y} \cdot \overrightarrow{\mathcal{E}}_{Y}$ (here $g^{\prime}$ is the hypercharge gauge coupling constant). Now, the presence of nonhomogeneous hypermagnetic fields in the EW plasma with (hyper)conductivity $\sigma_{c}$ always implies the existence of a related electric field, $\overrightarrow{\mathcal{E}}_{Y} \sim\left(1 / \sigma_{c}\right) \vec{\nabla} \times \overrightarrow{\mathcal{H}}_{Y}$. Since for a general stochastic magnetic background $\left\langle\left(\overrightarrow{\mathcal{H}}_{Y} \cdot \vec{\nabla} \times \overrightarrow{\mathcal{H}}_{Y}\right)^{2}\right\rangle \neq 0$, the nonuniform hypermagnetic field may absorb or release fermions and produce, ultimately, baryon and lepton density perturbations because of the anomaly equation.

The behavior of cold fermionic matter with nonzero anomalous Abelian charges was considered in [28] where it was pointed out that the anomalous fermionic matter is unstable against the creation of Abelian gauge field with nonzero Chern-Simons number, which eats up fermions because of the anomaly. It was suggested in [13] that the rightelectron number density may be converted to the hypercharge field because of a similar effect. The main aim of this paper is the study of the opposite situation, namely, we want to understand how hypercharge fields may be converted into fermions in a hot environment.

\section{B. MHD equations for ordinary plasmas}

During the symmetric phase of the electroweak theory the evolution of the background geometry is dominated by radiation. The first assumption we will make is that prior to $T_{c} \simeq 100 \mathrm{GeV}$ the geometry may be described by a conformally flat metric of the Friedmann-Robertson-Walker (FRW) type, whose line element is

$$
d s^{2}=g_{\alpha \beta} d x^{\alpha} d x^{\beta}=a(\tau)^{2}\left(d \tau^{2}-d \vec{x}^{2}\right), \quad a(\tau) \sim \tau
$$

[ $\tau$ is the conformal time coordinate related to the cosmic time coordinate $t$ as $d t=a(\tau) d \tau]$. We will also assume that the radiation-dominated stage started much before the electroweak epoch at temperatures $T>T_{c}$.

The Weyl invariance of the ordinary Maxwell equations in a conformally flat FRW background geometry implies that the MHD equations in the metric (2.1) can be written [29] as

$$
\begin{gathered}
\frac{\partial \vec{H}}{\partial \tau}=-\vec{\nabla} \times \vec{E}, \quad \frac{\partial \vec{E}}{\partial \tau}+\vec{J}=\vec{\nabla} \times \vec{H}, \\
\vec{\nabla} \cdot \vec{H}=0, \quad \vec{\nabla} \cdot \vec{E}=0,
\end{gathered}
$$

$$
\vec{\nabla} \cdot \vec{J}=0, \quad \vec{J}=\sigma(\vec{E}+\vec{v} \times \vec{H})
$$

$\left(\vec{H}=a^{2} \overrightarrow{\mathcal{H}}, \quad \vec{E}=a^{2} \overrightarrow{\mathcal{E}} ; \quad \vec{A}=a \overrightarrow{\mathcal{A}} ; \vec{J}=a^{3} \vec{J} ; \quad \sigma=\sigma_{c} a ; \overrightarrow{\mathcal{H}}=\vec{\nabla} \times \overrightarrow{\mathcal{A}} ;\right.$ $\overrightarrow{\mathcal{E}}=-\partial / \partial t \overrightarrow{\mathcal{A}} ; \overrightarrow{\mathcal{H}}, \overrightarrow{\mathcal{E}}, \overrightarrow{\mathcal{A}}, \vec{J}, \sigma_{c}$ are the flat-space quantities whereas $\vec{H}, \vec{E}, \vec{A}, \vec{J}, \sigma$ are the curved-space ones; $\vec{v}$ is the bulk velocity of the plasma). We assumed that the plasma is locally electrically neutral $(\vec{\nabla} \cdot \vec{E}=0)$ over length scales larger than the Debye radius. We notice that the spatial gradients used in Eq. (2.2) are defined according to the metric (2.1).

There are several approximations in which the above equations can be studied. One is the so-called ideal (or superconducting) approximation and the other is the real (or resistive) case $[25,30]$.

In the ideal case $\sigma^{-1}=0$ and, from Ohm's law we can immediately deduce that the electric field is orthogonal to the magnetic one and it is also orthogonal to the bulk velocity of the plasma:

$$
\vec{E} \simeq-\vec{v} \times \vec{H} .
$$

Two important theorems of the ideal MHD, which follow from Eq. (2.3), are the conservation of the magnetic flux:

$$
\Phi=\int_{\Sigma} \vec{H} \cdot d \vec{\Sigma}
$$

and of the magnetic helicity (Chern-Simons number) [2,30]:

$$
N_{\mathrm{CS}}=\frac{\alpha_{\mathrm{em}}}{\pi} \int_{V} d^{3} x \vec{H} \cdot \vec{A}, \quad \alpha_{\mathrm{em}}=\frac{e^{2}}{4 \pi},
$$

where $d \Sigma$ is a closed surface in the plasma; the volume integral is performed over a magnetic flux tube.

If, on the contrary, the effect of the finite value of the conductivity is taken into account $\left(\sigma^{-1} \ll 1\right)$ and the resistive $\mathrm{Ohm}$ law is employed, then the induced electric field is not exactly orthogonal to the magnetic one and the conservation laws of the ideal MHD are corrected (in the resistive approximation) by an expansion in powers of the resistivity, which can be explicitly computed and which we report at the lowest order in $\sigma^{-1}$ :

$$
\begin{aligned}
& \frac{d}{d \tau} \Phi=-\frac{1}{\sigma} \int_{\Sigma} \vec{\nabla} \times(\vec{\nabla} \times \vec{H}) \cdot d \vec{\Sigma}, \\
& \frac{d}{d \tau} N_{\mathrm{CS}}=-\frac{\alpha_{\mathrm{em}}}{\pi \sigma} \int_{V} d^{3} x \vec{H} \cdot \vec{\nabla} \times \vec{H} .
\end{aligned}
$$

According to Eqs. (2.6) the magnetic flux lines evolve glued to the hypercharged plasma element; also the sum of the link and twist number of the magnetic flux tubes is always the same all along the time evolution, only provided that $\sigma^{-1}$ $=0$.

The same dynamical information encoded in the magnetic flux conservation is also contained in the magnetic diffusivity equation, which can be derived according to Eq. (2.2):

$$
\frac{\partial \vec{H}}{\partial \tau}=\vec{\nabla} \times(\vec{v} \times \vec{H})+\frac{1}{\sigma} \nabla^{2} \vec{H} .
$$


The ratio of the two terms on the right-hand side defines the magnetic Reynolds number

$$
R \simeq \frac{\sigma|\bar{\nabla} \times \vec{v} \times \vec{H}|}{\left|\nabla^{2} \vec{H}\right|} \simeq v l \sigma .
$$

If $R \ll 1$ (for a given length scale $l$ ) the flux lines of the magnetic field will diffuse through the plasma. If $R \gg 1$ the flux lines of the magnetic field will be frozen into the plasma element. From the magnetic diffusivity equation (2.6) it is possible to derive the typical structure of the dynamo term by carefully averaging over the velocity field according to the procedure outlined in $[2,4]$. By assuming that the motion of the fluid is random and has zero mean velocity, it is possible to average over the ensemble of the possible velocity fields. In more physical terms this averaging procedure of Eq. (2.7) is equivalent to averaging over scales and times exceeding the characteristic correlation scale and time $\tau_{0}$ of the velocity field. This procedure assumes that the correlation scale of the magnetic field is much larger than the correlation scale of the velocity field. In this approximation the magnetic diffusivity equation can be written as

$$
\frac{\partial \vec{H}}{\partial \tau}=\alpha(\vec{\nabla} \times \vec{H})+\frac{1}{\sigma} \nabla^{2} \vec{H}
$$

$\left[\alpha=-\left(\tau_{0} / 3\right)\langle\vec{v} \cdot \vec{\nabla} \times \vec{v}\rangle\right.$ is the so-called dynamo term, which vanishes in the absence of vorticity; in this equation $\vec{H}$ is the magnetic field averaged over times larger than $\tau_{0}$, which is the typical correlation time of the velocity field]. We can clearly see that the crucial requirement for the all averaging procedure we described is that the turbulent velocity field has to be "globally" non-mirror-symmetric. It is interesting to point out [2] that the dynamo term in Eq. (2.9) has a simple electrodynamical meaning, namely, it can be interpreted as a mean Ohmic current directed along the magnetic field:

$$
\vec{J}=-\alpha \vec{H} .
$$

This equation tells us that an ensemble of screwlike vortices with zero mean helicity is able to generate loops in the magnetic flux tubes in a plane orthogonal to the one of the original field. This observation will be of some related interest for the physical interpretation of the results we are going to present in the following paragraph. We finally notice that if the velocity field is parity invariant (i.e., no vorticity for scales comparable with the correlation length of the magnetic field), then the dynamics of the infrared modes is decoupled from the velocity field since, over those scales, $\alpha$ $=0$.

\section{AMHD equations for electroweak plasmas}

The electroweak plasma in complete thermal equilibrium at a temperature $T$ can be characterized by $n_{f}$ chemical potentials $\mu_{i}, i=1, \ldots, n_{f}$ corresponding to the exactly conserved global charges

$$
N_{i}=L_{i}-\frac{B}{n_{f}}
$$

( $L_{i}$ is the lepton number of the $i$ th generation, $B$ is the baryon number, and $n_{f}$ is the number of fermionic generations). One should also introduce a chemical potential $\mu_{Y}$ corresponding to weak hypercharge, but its value is fixed from the requirement of the hypercharge neutrality of the plasma, $\langle Y\rangle=0$.

We want to study this plasma slightly out of thermal equilibrium; in particular, we want to see what happens with a nonuniform distribution of the hypermagnetic field. Because of the anomaly, and thanks to the arguments illustrated in Sec. II A, this field is coupled to the fermionic number densities. In principle, different chemical potentials can be assigned to all the fermionic degrees of freedom of the electroweak theory (45 if $n_{f}=3$ ) and the coupled system of Boltzmann-type equations for these chemical potentials and the hypercharge fields may be written. Since we are interested in the slow processes in the plasma, this is not necessary. If the coupling, corresponding to some slow process, is switched off, then the electroweak theory acquires an extra conserved charge and a further chemical potential should be added to the system given in Eq. (2.11).

An interesting observation (which turns out to be quite important in our context) has been made in [31], where it was noticed that perturbative reactions with right-handed electron chirality flip are out of thermal equilibrium at temperatures higher than some temperature ${ }^{1} T_{R}$. Thus the number of right electrons is perturbatively conserved at temperatures $T>T_{R}$ and the chemical potential $\mu_{R}$ can be introduced for it. On the other hand, this charge is not conserved because of the Abelian anomaly,

$$
\partial_{\mu} j_{R}^{\mu}=-\frac{g^{\prime 2} y_{R}^{2}}{64 \pi^{2}} \mathcal{Y}_{\mu \nu} \widetilde{\mathcal{Y}}^{\mu \nu},
$$

and it is therefore coupled to the hypermagnetic field. Here $\mathcal{Y}$ and $\widetilde{\mathcal{Y}}$ are, respectively, the $\mathrm{U}_{Y}(1)$ hypercharge field strengths and their duals, $g^{\prime}$ is the associated gauge coupling, and $y_{R}=-2$ is the hypercharge of the right electron.

Now we are ready to derive the anomalous MHD equations $[13,26]$. The effective Lagrangian density describing the dynamics of the gauge fields at finite fermionic density is [32]

$$
\begin{gathered}
\mathcal{L}_{Y, e_{R}}=-\frac{1}{4} \sqrt{-g} Y_{\alpha \beta} Y^{\alpha \beta}-\sqrt{-g} J_{\alpha} Y^{\alpha}+\mu \epsilon_{i j k} Y^{i j} Y^{k}, \\
\mu=\frac{g^{\prime 2}}{4 \pi^{2}} \mu_{R}
\end{gathered}
$$

( $g$ is the determinant of the metric defined in Eq. (2.1); $Y_{\alpha \beta}=\nabla_{[\alpha} Y_{\beta]} ; \nabla_{\alpha}$ is the covariant derivative with respect to the metric (2.1) [notice that in the metric (2.1) $\nabla_{[\alpha} Y_{\beta]}$ $\left.=\partial_{[\alpha} Y_{\beta]}\right] ; g^{\prime}$ is the Abelian coupling constant). The first and the last terms in Eq. (2.13) are nothing but the curved-space generalization of the flat-space effective Lagrangian for the hypercharge fields at finite fermion density [32], $J_{\alpha}$ is the

\footnotetext{
${ }^{1}$ This temperature depends on the particle physics model, see also the discussion reported in Sec. V. In the MSM $T_{R} \simeq 80 \mathrm{TeV}$ [31].
} 
Ohmic current. The equations of motion for the hyperelectric and hypermagnetic fields are then

$$
\begin{gathered}
\frac{\partial \vec{H}_{Y}}{\partial \tau}=-\vec{\nabla} \times \vec{E}_{Y}, \quad \frac{\partial \vec{E}_{Y}}{\partial \tau}+\vec{J}_{Y}=\frac{g^{\prime 2}}{\pi^{2}} \mu_{R} a \vec{H}_{Y}+\vec{\nabla} \times \vec{H}_{Y}, \\
\vec{\nabla} \cdot \vec{H}_{Y}=0, \quad \vec{\nabla} \cdot \vec{E}_{Y}=0, \\
\vec{\nabla} \cdot \vec{J}_{Y}=0, \quad \vec{J}_{Y}=\sigma\left(\vec{E}_{Y}+\vec{v} \times \vec{H}_{Y}\right), \quad \sigma=\sigma_{c} a(\tau)
\end{gathered}
$$

$\left[\vec{E}_{Y}=a^{2} \overrightarrow{\mathcal{E}}_{Y}, \vec{H}_{Y}=a^{2} \overrightarrow{\mathcal{H}}_{Y}, \overrightarrow{\mathcal{H}}_{Y}=\vec{\nabla} \times \overrightarrow{\mathcal{Y}}, \overrightarrow{\mathcal{E}}_{Y}=-(\partial / \partial t) \overrightarrow{\mathcal{Y}}\right]$. For the EW theory the conductivity of plasma is $\sigma \simeq 70 T$ [33]. To the equations of motion of the hypercharge field (2.14) we have to add the evolution equation of the right-electron chemical potential, which accounts for the anomalous and perturbative nonconservation of the right-electron number density $\left(n_{R}\right)$ :

$$
\frac{\partial n_{R}}{\partial t}=-\frac{g^{\prime 2}}{4 \pi^{2}} \overrightarrow{\mathcal{E}}_{Y} \cdot \overrightarrow{\mathcal{H}}_{Y}-\Gamma\left(n_{R}-n_{R}^{\mathrm{eq}}\right),
$$

where $\Gamma$ is the perturbative chirality-changing rate, $\Gamma$ $=T\left(T_{R} / M_{0}\right), n_{R}^{\mathrm{eq}}$ is the equilibrium value of the rightelectron number density, and the term proportional to $\vec{E}_{Y} \cdot \vec{H}_{Y}$ is the right-electron anomaly contribution.

Finally, the relationship between the right-electron number density and the chemical potential must be specified. This relation depends upon the particle content of the theory, e.g., upon the number of fermionic generations, the number of Higgs doublets, etc. We will write it for the case of the MSM [34]: the specific coefficients change only slightly for other theories and do not have a significant impact on the results. For generality, we assume that the universe is asymmetric not only with respect to the number of right electrons, but also with respect to corresponding conserved charges defined in Eq. (2.11) and compute all the relevant chemical potentials:

$$
\begin{aligned}
& \mu_{R}=\frac{2}{45} \pi^{2} N_{\text {eff }}\left(\frac{783}{88} \delta_{R}-\frac{201}{88} \delta_{1}+\frac{15}{22}\left(\delta_{2}+\delta_{3}\right)\right) T, \\
& \mu_{1}=\frac{2}{45} \pi^{2} N_{\text {eff }}\left(-\frac{201}{88} \delta_{R}+\frac{1227}{440} \delta_{1}+\frac{3}{110}\left(\delta_{2}+\delta_{3}\right)\right) T, \\
& \mu_{2}=\frac{2}{45} \pi^{2} N_{\text {eff }}\left(\frac{15}{22} \delta_{R}+\frac{3}{110} \delta_{1}+\frac{14}{55} \delta_{2}+\frac{124}{55} \delta_{3}\right) T, \\
& \mu_{3}=\frac{2}{45} \pi^{2} N_{\text {eff }}\left(\frac{15}{22} \delta_{R}+\frac{3}{110} \delta_{1}+\frac{14}{55} \delta_{2}+\frac{124}{55} \delta_{3}\right) T, \\
& \mu_{Y}=\frac{2}{45} \pi^{2} N_{\text {eff }}\left(\frac{27}{88} \delta_{R}+\frac{11}{440} \delta_{1}+\frac{39}{110}\left(\delta_{2}+\delta_{3}\right)\right) T,
\end{aligned}
$$

where $\delta_{i}$ is the asymmetry of $i$ th conserved charge, $\delta_{R}$ $=n_{R} / s$ ( $s$ is the entropy density) is the right-electron asymmetry, and $N_{\text {eff }}=106.75$ is the effective number of relativistic degrees of freedom in the symmetric phase of the MSM.
With the use of relations (2.16), Eq. (2.15) can be rewritten completely in terms of the right-electron chemical potential:

$$
\frac{1}{a} \frac{\partial\left(\mu_{R} a\right)}{\partial \tau}=-\frac{g^{\prime 2}}{4 \pi^{2}} \frac{783}{88} \frac{1}{a^{3} T^{2}} \vec{E}_{Y} \cdot \vec{H}_{Y}-\Gamma\left(\mu_{R} a\right) .
$$

At finite hyperconductivity (in what we would call, in a MHD context, "resistive" approximation) we have that from Eq. (2.14) the induced hyperelectric field is not exactly orthogonal to the hypermagnetic one and, moreover, an extra "fermionic" current comes into the game thanks to the fact that we are working at finite chemical potential. Therefore in our context the resistive Ohm law can be written as

$$
\begin{aligned}
\vec{E}_{Y}= & \frac{\vec{J}_{Y}}{\sigma}-\vec{v} \times \vec{H}_{Y} \simeq \frac{1}{\sigma}\left(\frac{4 \alpha^{\prime}}{\pi} \mu_{R} a \vec{H}_{Y}+\vec{\nabla} \times \vec{H}_{Y}\right) \\
& -\vec{v} \times \vec{H}_{Y}, \quad \alpha^{\prime}=\frac{g^{\prime 2}}{4 \pi} .
\end{aligned}
$$

In the parentheses appearing in Eq. (2.18) we can identify two different contributions. One is associated with the curl of the magnetic field. We will call this the MHD contribution, since it appears in the same way in ordinary plasmas. The other contribution contains the chemical potential and it is directly proportional to the magnetic field and to the chemical potential. This is a typical finite density effect. In fact the extra Ohmic current simply describes the possibility that the energy sitting in real fermionic degrees of freedom can be transferred to the hypermagnetic field. It may be of some interest to notice the analogy between the first term of Eq. (2.18) and the typical form of the Ohmic current discussed in Eq. (2.10) appearing in the context of the dynamo mechanism. In the latter case the presence of a current (proportional to the vorticity through the $\alpha$ dynamo term) was indicating that large length scale magnetic fields could grow by eating up fluid vortices. By inserting $\vec{E}_{Y}$ obtained from the generalized $\mathrm{Ohm}$ law (2.18) in the evolution equations (2.14) of the hypercharge fields, we obtain the generalized form of the magnetic diffusivity equation (2.7):

$$
\frac{\partial \vec{H}_{Y}}{\partial \tau}=-\frac{4 a \alpha^{\prime}}{\pi \sigma} \vec{\nabla} \times\left(\mu_{R} \vec{H}_{Y}\right)+\vec{\nabla} \times(\vec{v} \times \vec{H})+\frac{1}{\sigma} \nabla^{2} \vec{H}_{Y} .
$$

In order to be consistent with our resistive approach we neglected terms containing time derivatives of the electric field, which are subleading provided the conductivity is finite. In our considerations we will also make a further simplification, namely, we will assume that the EW plasma is (globally) parity invariant and that, therefore, no global vorticity is present. Therefore, since the length scale of variation of the bulk velocity field is much shorter than the correlation distance of the hypermagnetic field, the infrared modes of the hypercharge will be practically unaffected by the velocity of the plasma, which will be neglected when the large-scale part of the hypercharge is concerned. This corresponds to the usual MHD treatment of a mirror-symmetric plasma [see, e.g., Eq. (2.9), when $\alpha=0$ ]. 
Equations (2.19) and (2.17) form a set of AMHD equations for the hypercharge magnetic field and right-electron chemical potential in the expanding universe.

An important property of Eqs. (2.14) and (2.19) is that they are not conformally invariant. The conformal invariance of the ordinary Maxwell equations implies that the equations for the rescaled fields in curved space keep the same form also in flat space in terms of the nonrescaled fields provided the conformal time coordinate is employed in curved space and the cosmic time coordinate is employed in flat space. We can easily see that this is not the case of Eq. (2.14) by writing our evolution equations in flat space:

$$
\begin{aligned}
\frac{\partial \overrightarrow{\mathcal{H}}_{Y}}{\partial t}=-\vec{\nabla} \times \overrightarrow{\mathcal{E}}_{Y}, \quad \frac{\partial \overrightarrow{\mathcal{E}}_{Y}}{\partial t}+\vec{J}_{Y}=\frac{g^{\prime 2}}{\pi^{2}} \mu_{R} \overrightarrow{\mathcal{H}}_{Y}+\vec{\nabla} \times \overrightarrow{\mathcal{H}}_{Y}, \\
\vec{\nabla} \cdot \overrightarrow{\mathcal{H}}_{Y}=0, \quad \vec{\nabla} \cdot \overrightarrow{\mathcal{E}}_{Y}=0 \\
\vec{\nabla} \cdot \vec{J}_{Y}=0, \quad \vec{J}_{Y}=\sigma_{c} \overrightarrow{\mathcal{E}}_{Y} .
\end{aligned}
$$

The lack of conformal invariance comes from the presence of the scale factor in front of the right-electron chemical potential in the evolution equation (2.14) for $\vec{H}_{Y}$. Clearly, the explicit breaking of conformal invariance is also reflected in the Ohm law and in the hypermagnetic diffusivity equation which, passing from curved to flat space, become

$$
\begin{gathered}
\overrightarrow{\mathcal{E}}_{Y}=\frac{\vec{J}_{Y}}{\sigma_{c}} \simeq \frac{1}{\sigma_{c}}\left(\frac{\alpha^{\prime}}{\pi} \mu_{R} \overrightarrow{\mathcal{H}}_{Y}+\vec{\nabla} \times \overrightarrow{\mathcal{H}}_{Y}\right), \\
\frac{\partial \overrightarrow{\mathcal{H}}_{Y}}{\partial t}=-\frac{4 \alpha^{\prime}}{\pi \sigma} \vec{\nabla} \times\left(\mu_{R} \overrightarrow{\mathcal{H}}_{Y}\right)+\frac{1}{\sigma_{c}} \nabla^{2} \overrightarrow{\mathcal{H}}_{Y} .
\end{gathered}
$$

In flat space the kinetic equation of the right-electron chemical potential becomes instead

$$
\frac{\partial \mu_{R}}{\partial t}=-\frac{g^{\prime 2}}{4 \pi^{2} T^{2}} \frac{783}{88} \overrightarrow{\mathcal{E}}_{Y} \cdot \overrightarrow{\mathcal{H}}_{Y}-\Gamma \mu_{R}
$$

It is interesting to notice that the term containing the chemical potential in Eq. (2.19) plays a role similar to that of the dynamo term, since it also produces an instability [13]. Its physical interpretation is actually quite simple. We could define, as in the case of the ordinary MHD, a generalized Reynolds number that measures the relative weight of the two terms on the right-hand side of Eq. (2.19). If the diffusion term dominates, then the flux of magnetic hypercharge will diffuse through the plasma. If on the contrary we are in the inertial range (where the diffusivity term is negligible) there are two possibilities. If the chemical potential is exactly zero then the hypermagnetic field will be frozen into the plasma element as required by magnetic flux conservation. However, if we are at finite fermion density the energy density sitting in fermionic degrees of freedom may be transformed in infrared modes of the hypermagnetic field.

\section{FERMIONS FROM THE HYPERCHARGE FIELD}

\section{A. $T>T_{c}$}

In this section we are going to compute the relationship between hypermagnetic fields and induced fermionic chemical potential, at temperatures larger than the critical temperature of the electroweak phase transition $T_{c}$.

There is an important consequence of the resistive approximation. By using the Ohm law given by Eq. (2.21) we can eliminate the hyperelectric field from the kinetic equation of the right electrons and obtain

$$
\frac{\partial}{\partial t}\left(\frac{\mu_{R}}{T}\right)=-\frac{g^{\prime 2}}{4 \pi^{2} \sigma_{c} T^{3}} \frac{783}{88} \overrightarrow{\mathcal{H}}_{Y} \cdot \vec{\nabla} \times \overrightarrow{\mathcal{H}}_{Y}-\left(\Gamma+\Gamma_{\mathcal{H}}\right) \frac{\mu_{R}}{T},
$$

where

$$
\Gamma_{\mathcal{H}}=\frac{783}{22} \frac{\alpha^{\prime 2}}{\sigma_{c} \pi^{2}} \frac{\left|\overrightarrow{\mathcal{H}}_{Y}\right|^{2}}{T^{2}}
$$

We notice immediately that the source term appearing in the right-hand side of Eq. (3.1) (and coming from the anomaly) is indeed strongly reminiscent of what we would call (in a MHD context) magnetic helicity. From Eq. (3.1) one can see that the right-electron number is not conserved (even if $\Gamma$ $=0$ ) because of the Abelian anomaly, provided a nonzero hypermagnetic field is present (cf. Ref. [35]). Equation (3.1) can be solved in the adiabatic approximation at temperatures $T<T_{R}$, when perturbative right-electron chirality flip reactions are in thermal equilibrium. Neglecting the time derivative of the chemical potential, we get

$$
\frac{\mu_{R}}{T} \simeq-\frac{\alpha^{\prime}}{\pi \sigma_{c} T^{3}} \frac{783}{88} \frac{\overrightarrow{\mathcal{H}}_{Y} \cdot \vec{\nabla} \times \overrightarrow{\mathcal{H}}_{Y}}{\Gamma+\Gamma_{\mathcal{H}}} .
$$

The solution (3.3) can be inserted into Eq. (2.22) for the magnetic field, which will become a partial (nonlinear) differential equation containing only the hypermagnetic field. Thus an inhomogeneous hypermagnetic field can produce a spatial variation in the chemical content of the plasma. In fact, according to Eq. (2.16), spatial fluctuations in the rightelectron chemical potential will determine fluctuations not only in the right-electron number density but also in the number densities associated with the other fermion asymmetries. Fluctuations in the number density of some species are frequently called isocurvature perturbations. There are actually two regimes where Eq. (3.3) can be analyzed. The first one is the regime where $\Gamma>\Gamma_{\mathcal{H}}$. In this case the rate of right-electron dilution is essentially determined by the perturbative processes, which can flip the chirality of the right electrons. In the opposite case $\left(\Gamma_{\mathcal{H}}>\Gamma\right)$ the rate is mainly due to the presence of the Abelian anomaly, and

$$
n_{R} \simeq-\frac{88 \pi^{2}}{783 g^{\prime 2}}\left(\frac{\overline{\mathcal{H}}_{Y} \cdot \bar{\nabla} \times \overline{\mathcal{H}}_{Y}}{\left|\overline{\mathcal{H}}_{Y}\right|^{2}}\right)+O\left(\frac{\Gamma}{\Gamma_{\mathcal{H}}}\right), \quad \Gamma_{\mathcal{H}}>\Gamma .
$$

In Eq. (3.4) the chirality-changing rate only comes in the correction. Moreover, since the hypermagnetic field intensity appears with the same power in the numerator and in the 
denominator, the right-electron fluctuations are independent of the magnitude of the hypermagnetic field fluctuations and fixed by their spatial distribution. It is interesting to notice that in Eq. (3.4) the actual value of the conductivity completely cancels and only appears in the correction.

\section{B. $T<T_{c}$}

Now we are going to discuss what happens after the electroweak phase transition. Below $T_{c}$ the hypercharge magnetic fields are converted into ordinary magnetic fields. The latter do not have anomalous coupling to fermions, and the usual MHD equations are fully valid. Any source term that was inducing a nonvanishing chemical potential disappears. Thus the transformation of the magnetic fields into fermions is no longer possible. It seems, therefore, that the matter fluctuations after the phase transition will be given by the fluctuations right before the phase transition. The last statement is in fact wrong for two reasons. First, if the phase transition is weakly first order, so that sphaleron processes are in thermal equilibrium after it, then any fluctuations of the fermionic charges will disappear. In this particular case all anomalous effects that existed before $T_{c}$ are simply "forgotten," since the system passes through an equilibrium period with respect to fermion-number nonconservation. Let us admit that the electroweak phase transition is strongly first order and a necessary condition for EW baryogenesis [36] is satisfied. Then, there is an important "storage", effect (and we come to the second point), which amplifies the estimates of Eqs. (3.3) and (3.4) by many orders of magnitude. The point is that the fermion number can sit not only in the fermions (and in their associated chemical potential), but also in the hypermagnetic field itself. At the EW phase transition, this fermion number must be released in the form of real fermions, just because the ordinary magnetic field, which survives after transition, cannot carry fermion number. To compute the density of the Chern-Simons number $n_{\mathrm{CS}}$ of the hypercharge field configuration before the EW phase transition we just integrate $\overrightarrow{\mathcal{E}}_{Y} \cdot \overrightarrow{\mathcal{H}}_{Y}$ over the time:

$$
\Delta n_{\mathrm{CS}}\left(t_{c}\right)=-\frac{y_{R}^{2} g^{\prime 2}}{16 \pi^{2}} \int_{0}^{t_{c}} \overrightarrow{\mathcal{E}}_{Y} \cdot \overrightarrow{\mathcal{H}}_{Y} d t .
$$

In order to estimate this integral we have to solve the coupled system given by Eqs. (2.22) and (3.1). The main contribution to this integral comes from the largest time $t$ $\sim t_{c}$, where reactions with right-electron chirality flip are in thermal equilibrium. Thus we can use again the adiabatic approximation (which implies that $\partial \mu_{R} / \partial t \sim 0$ ) and obtain

$$
\begin{gathered}
\Delta n_{\mathrm{CS}}\left(t_{c}\right)=-\frac{\alpha^{\prime}}{2 \pi} \int_{0}^{t_{c}} \frac{\Lambda(\vec{x}, t)}{\Gamma+\Gamma_{\mathcal{H}}} \frac{\Gamma}{\sigma_{c}} d t, \\
\Lambda(\vec{x}, t)=\overrightarrow{\mathcal{H}}_{Y} \cdot \vec{\nabla} \times \overrightarrow{\mathcal{H}}_{Y} .
\end{gathered}
$$

This Chern-Simons number will be released at the EW phase transition in the form of fermions, which will not be destroyed by the sphalerons if the phase transition is strongly first order. The density of the baryonic number $n_{B}$ is just given by integrated anomaly:

$$
n_{B}\left(t_{c}\right)=-\frac{n_{f}}{2} \Delta n_{\mathrm{CS}}\left(t_{c}\right)
$$

This equation is our main result. Once the hypermagnetic background is specified, the time integration appearing in Eq. (3.6) can be performed. If the typical scale of the configuration is larger than the magnetic diffusivity distance

$$
r_{\sigma} \sim \frac{1}{k_{\sigma}} \sim 10^{-9} \times L_{E W}
$$

where $L_{E W} \sim 3 \mathrm{~cm}$ is the size of the $\mathrm{EW}$ horizon at $T_{c}$ $\sim 100 \mathrm{GeV}$, then all the modes of the hypermagnetic field with momentum $k$ smaller than

$$
k_{\sigma} \sim \sqrt{\frac{\sigma_{c}}{M_{0}}} T, \quad M_{0}=\frac{M_{P l}}{1.66 \sqrt{N_{\text {eff }}}} \sim 7.1 \times 10^{17} \mathrm{GeV}
$$

will remain frozen into the EW plasma element. Thus the baryon-number fluctuations can be written as

$$
\begin{gathered}
\delta\left(\frac{n_{B}}{s}\right)\left(\vec{x}, t_{c}\right)=\frac{\alpha^{\prime}}{2 \pi \sigma_{c}} \frac{n_{f}}{s} \frac{\overrightarrow{\mathcal{H}}_{Y} \cdot \vec{\nabla} \times \overrightarrow{\mathcal{H}}_{Y}}{\Gamma+\Gamma_{\mathcal{H}}} \frac{\Gamma M_{0}}{T_{c}^{2}}, \\
\Gamma_{\mathcal{H}}=\frac{783}{22} \frac{\alpha^{\prime 2}}{\sigma_{c} \pi^{2}} \frac{\left|\overrightarrow{\mathcal{H}}_{Y}\right|^{2}}{T_{c}^{2}} .
\end{gathered}
$$

Notice that in Eq. (3.9) there is an enhancement by a factor $\sim \Gamma M_{0} / T_{c}^{2}$ arising from the time integration of the anomaly term. We also point out that for $\Gamma_{\mathcal{H}} \lesssim \Gamma$ the rate of rightelectron chirality flip cancels out. This last expression can be easily written in terms of the corresponding curved space quantities and the only point to be kept in mind is that, in curved-space, the chemical potential is multiplied by the scale factor (which breaks the conformal invariance of the AMHD equations).

\section{STOCHASTIC HYPERMAGNETIC BACKGROUNDS}

Two qualitatively different classes of hypermagnetic backgrounds can be studied. The first class is characterized by a nonvanishing magnetic helicity [i.e., $\left\langle\overrightarrow{\mathcal{H}}_{Y} \cdot \vec{\nabla} \times \overrightarrow{\mathcal{H}}_{Y}\right\rangle$ $\neq 0]$, which implies that the hypercharge field is topologically nontrivial and parity noninvariant. Therefore, in this class of backgrounds not only fluctuations in the baryon number will be produced, but also the generation of the baryon asymmetry is possible. We will discuss this possibility later in Sec. VI.

The aim of this section is to relate the properties of stochastic background with zero average magnetic helicity to the baryon-number fluctuations. For this type of magnetic field $\left\langle\delta\left(n_{B} / s\right)\left(\vec{x}, t_{c}\right)\right\rangle=0$ but $\left\langle\delta\left(n_{B} / s\right)\left(\vec{x}+\vec{r}, t_{c}\right) \delta\left(n_{B} /\right.\right.$ $\left.s)\left(\vec{x}, t_{c}\right)\right\rangle \neq 0$, so that only the inhomogeneities of baryonic number are produced. We will be interested here in the formal aspect of this relation, and we will focus our attention on the study of the phenomenological applications in Sec. V.

Consider a stochastic hypermagnetic field whose (parityinvariant) two-point function is 


$$
G_{i j}(\vec{r})=\left\langle H_{i}(\vec{x}) H_{j}(\vec{x}+\vec{r})\right\rangle=\int e^{i \vec{k} \cdot \vec{r}} G_{i j}(k) d^{3} k,
$$

where, because of transversality of the magnetic field,

$$
G_{i j}(k)=k^{2} f(k)\left(\delta_{i j}-\frac{k_{i} k_{j}}{k^{2}}\right) \text {. }
$$

The average appearing in Eq. (4.1) denotes an ensemble average. As was previously stated, the average hypermagnetic helicity is $\left\langle\Lambda\left(\vec{x}, t_{c}\right)\right\rangle=\langle\vec{H} \cdot \vec{\nabla} \times \vec{H}\rangle=0$ in the case of the transverse and parity-invariant two-point function given in Eqs. (4.1) and (4.2).

The assumption of the stochasticity of the background implies that the higher-order correlation functions of the magnetic hypercharge fields can be computed in terms of the two-point function (4.1). For example, the four-point function can be completely expressed in terms of the two-point function (4.1):

$$
\begin{aligned}
\left\langle H_{k}\left(\vec{x}^{\prime}\right) H_{j}(\vec{x}) H_{l}\left(\vec{y}^{\prime}\right) H_{n}(\vec{y})\right\rangle & \\
= & {\left[\left\langle H_{k}\left(\vec{x}^{\prime}\right) H_{j}(\vec{x})\right\rangle\left\langle H_{l}\left(\vec{y}^{\prime}\right) H_{n}(\vec{y})\right\rangle+\left\langle H_{k}\left(\vec{x}^{\prime}\right) H_{l}\left(\vec{y}^{\prime}\right)\right\rangle\right.} \\
& \left.\times\left\langle H_{j}(\vec{x}) H_{n}(\vec{y})\right\rangle+\left\langle H_{k}\left(\vec{x}^{\prime}\right) H_{n}(\vec{y})\right\rangle\left\langle H_{j}(\vec{x}) H_{l}\left(\vec{y}^{\prime}\right)\right\rangle\right] .
\end{aligned}
$$

We are now going to compute the level of the induced fluctuations by the above-mentioned stochastic hypermagnetic background. We parametrize the spectral properties of our correlation function by assuming a power law behavior of its Fourier transform:

$$
f(k)=\frac{1}{k}\left(\frac{k}{k_{1}}\right)^{\alpha} \exp \left[-2\left(\frac{k}{k_{\sigma}}\right)^{2}\right]
$$

This representation only depends upon two unknown parameters, namely, the slope $(\alpha)$ and the amplitude, which can be changed by changing $k_{1}$. The exponential damping appearing in the mode function is not the result of any assumption, but it is a direct consequence of the fact that, according to the hyperdiffusivity equations (2.19) and (2.22), all the modes $k>k_{\sigma}$ decay thanks to the finite value of the conductivity. The hypermagnetic energy density is obtained by tracing the Green function defined in Eq. (4.1) for $\vec{r}=0$,

$$
\left\langle|\vec{H}(\vec{x})|^{2}\right\rangle=\operatorname{Tr}\left[G_{i j}(0)\right]=2 \int d^{3} k k\left(\frac{k}{k_{1}}\right)^{\alpha} \exp \left[-2\left(\frac{k}{k_{\sigma}}\right)^{2}\right] .
$$

Because of the exponential damping, this integral is always ultraviolet convergent and can be very simply performed:

$$
\left\langle\frac{|\vec{H}(\vec{x})|^{2}}{T^{4}}\right\rangle=4 \pi \xi^{-\alpha} 2^{-(\alpha / 2+2)} \Gamma\left(\frac{\alpha+4}{2}\right)\left(\frac{k_{\sigma}}{T}\right)^{\alpha+4},
$$

where $\xi=k_{1} / T$.

We will often have to compute various four-point functions, and it is sometimes of great help to evaluate the higher-order Green functions not in Fourier space (where complicated convolutions would appear) but directly in real space. A generic rotationally and parity-invariant Green function can always be written in real space as

$$
G_{i j}(|\vec{r}|)=F_{1}(r) \delta_{i j}+r_{i} r_{j} F_{2}(r),|\vec{r}|=r
$$

where

$$
\begin{gathered}
F_{1}(r)=\frac{\partial}{\partial r^{2}}\left[r^{2} h\left(r^{2}\right)\right], \quad F_{2}(r)=-\frac{\partial}{\partial r^{2}}\left[h\left(r^{2}\right)\right], \\
r \mathcal{G}(r)=\frac{\partial}{\partial r^{2}}\left[r^{3} h\left(r^{2}\right)\right],
\end{gathered}
$$

and $\mathcal{G}(r)$ is nothing but the trace of our two-point function, namely,

$$
\mathcal{G}(r)=\operatorname{Tr}\left[G_{i j}(r)\right]=\frac{4 \pi}{R} k_{\sigma}^{4}\left(\frac{k_{\sigma}}{k_{1}}\right)^{\alpha} \int_{0}^{\infty} q^{(2+\alpha)} e^{-2 q^{2}} \sin R q d q
$$

(with $q=k / k_{\sigma}$ and $\left.R=r / r_{\sigma}\right)$. Clearly, this representation is transverse [i.e., $\left(\partial / \partial r^{i}\right) / G_{i j}(r)=0$ ], and moreover the integral over the spectrum appearing in Eq. (4.9) can be exactly performed in terms of known special functions:

$$
\begin{gathered}
\mathcal{G}(R)=\mathcal{G}(0) F\left(-\frac{1}{2}-\frac{\alpha}{2}, \frac{3}{2}, \frac{R^{2}}{8}\right) \exp \left[-\frac{R^{2}}{8}\right], \\
\mathcal{G}(0)=4 \pi k_{\sigma}^{4}\left(\frac{k}{k_{\sigma}}\right)^{\alpha} 2^{-3-\alpha / 2} \Gamma\left(2+\frac{\alpha}{2}\right)
\end{gathered}
$$

$[F(a, b, z)$ is the confluent hypergeometric function and $\Gamma(z)$ is the Euler gamma function [37,38]].

Some physical considerations are now in order. In our problem the relevant scales are those that are not erased by the plasma conductivity, namely, from Eq. (3.8), all the scales $r>r_{\sigma}$. Therefore the physical limit of all our correlation functions will always be the large- $R$ limit. Moreover, a physically realistic situation does correspond, in our considerations, to the case where the Green functions are decaying at large length scales. If the Green functions decay at large distances we automatically exclude the possibility that the energy spectrum of the hypermagnetic inhomogeneities will have some peak at large wavelength. The large-scale (i.e., $R>1$ ) limit of the normalized trace of our Green functions will then be given by

$$
g(R)=\frac{\Gamma\left(\frac{3}{2}\right)}{\Gamma(-(1+\alpha) / 2)} 2^{6+(3 / 2) \alpha} R^{-(\alpha+4)}, \quad g(R)=\frac{\mathcal{G}(R)}{\mathcal{G}(0)} .
$$

In $k$ space the magnetic energy density per logarithmic interval of frequency is defined as $[16,18] \rho(k)=d \rho_{H} / d \ln k$ [where $\rho_{H}=\frac{1}{2}\left\langle|\vec{H}(\vec{x})|^{2}\right\rangle$. Therefore in our case $\rho(k)$ $\sim k^{4}\left(k / k_{1}\right)^{\alpha}$ which implies that "blue" $(\alpha \gtrsim-4)$ or "violet' $(\alpha \gg-4)$ logarithmic energy spectra correspond to the physically interesting case of two-point functions decaying at large scales whereas for $\alpha<-4$ we have "red" logarithmic energy spectra which are connected with Green's functions 
decreasing at small scales. The case of flat logarithmic energy spectra $(\alpha \simeq-4)$ may quite naturally appear in string cosmological models [18].

It is important to point out that if the Green functions decay at large distances, then $g(R)<1$. This observation will be of some relevance when we will have to explicitly evaluate our fluctuations, since $g(R)$ will turn out to be a useful and natural expansion parameter (see Appendix B for details).

We can also give the large- $R$ expression of $h\left(r^{2}\right)$ since it can easily be deduced according to Eq. (4.8):

$$
h(R)=\mathcal{G}(0) \frac{\Gamma\left(\frac{3}{2}\right)}{\Gamma((3-\epsilon) / 2)} \frac{2^{(3 / 2) \epsilon+1}}{3-\epsilon} R^{-\epsilon},
$$

where we defined $\alpha=-4+\epsilon$.

We are now ready to compute the level of baryon-number fluctuations induced by our stochastic background of magnetic hypercharge fields, which is defined by the correlation function

$$
\Delta\left(r, \tau_{c}\right)=\sqrt{\mid\left\langle\delta\left(\frac{n_{B}}{s}\right)\left(\bar{x}, t_{c}\right) \delta\left(\frac{n_{B}}{s}\right)\left(\bar{x}+\bar{r}, t_{c}\right)\right\rangle} .
$$

There are two regimes where this calculation can be performed depending upon the relative weight of the two rates appearing in Eq. (3.1). If $\Gamma \geq \Gamma_{\mathcal{H}}$ the major technical problem we have to face is to evaluate the correlation function of the magnetic helicity at two different points; this involves, ultimately, the calculation of a four-point function. The algebraic details of this long but straightforward calculation are given in Appendix A. The result is given in terms of the functions appearing in the real space parametrization of our Green's functions given in Eq. (4.8):

$$
\begin{aligned}
&\langle(\vec{H} \cdot \vec{\nabla} \times \vec{H})(\vec{x})(\vec{H} \cdot \vec{\nabla} \times \vec{H})(\vec{x}+\vec{r})\rangle \\
&=-\frac{4}{r} F_{1}(r) \frac{d F_{2}(r)}{d r}-2 F_{1}(r) \frac{d^{2} F_{1}(r)}{d r^{2}} \\
&+4 r^{2}\left[F_{2}(r)\right]^{2}+2 r F_{1}(r) \frac{d F_{2}(r)}{d r} \\
&-6 r F_{2}(r) \frac{d F_{1}(r)}{d r}+6 F_{1}(r) F_{2}(r)+2\left(\frac{d F_{1}(r)}{d r}\right)^{2} .
\end{aligned}
$$

In this and the following formulas we will often use the notation $\vec{B}\left(\tau_{c}\right)=\vec{H}\left(\tau_{c}\right) / T^{2}\left(\tau_{c}\right)$, which is convenient since in $\vec{B}$ the time dependence of the scale factors cancels and the only time dependence left is due to the evolution of the effective number of the relativistic degrees of freedom in the plasma, $N_{\text {eff }}(\tau)$. Inserting now Eq. (4.12) into Eq. (4.8) we obtain

$$
\begin{gathered}
\Delta\left(r, t_{c}\right)=\frac{45 n_{f} \alpha^{\prime}}{\pi^{2} N_{\mathrm{eff}}\left(\tau_{c}\right)} \frac{T_{c}}{\sigma_{c}} \frac{M_{0}}{T_{c}} \frac{\xi^{4-\epsilon} C(\epsilon)}{\left(r T_{c}\right)^{1+\epsilon}}[1+O(\lambda)], \\
C(\epsilon)=\frac{2^{\epsilon-3 / 2} \Gamma\left(\frac{\epsilon}{2}\right)}{\Gamma((3-\epsilon) / 2)} \sqrt{\frac{\pi \epsilon(\epsilon+2)}{(3-\epsilon)}} \\
\xi=\frac{k_{1}}{T}, \quad \lambda \sim\left(\frac{\Gamma}{\Gamma_{\mathcal{H}}}\right)^{2}\left(\frac{r}{r_{\sigma}}\right)^{-2 \epsilon} .
\end{gathered}
$$

Notice that this expression holds for large rate (i.e., $\Gamma>\Gamma_{\mathcal{H}}$ ) and for large scales [i.e., $g(R)<1, R>1$ ].

The second regime in which one may wish to compute the level of induced fluctuations is the one where $\Gamma \leq \Gamma_{\mathcal{H}}$. The main mathematical problem will be to evaluate the correlation function of the hypermagnetic helicity divided by the hypermagnetic energy density. This is of course a strongly nonlinear object which we cannot compute exactly. By working at large scales and in the hypothesis that the Green functions are decaying at large distances, the bottom line of this calculation (reported in Appendix B) is

$$
\begin{aligned}
& \left\langle\left(\frac{\vec{B} \cdot \vec{\nabla} \times \vec{B}}{|\vec{B}|^{2}}\right)(\bar{x})\left(\frac{\vec{B} \cdot \bar{\nabla} \times \bar{B}}{|\vec{B}|^{2}}\right)(\vec{x}+\vec{r})\right\rangle \\
& \simeq \frac{\langle(\vec{B} \cdot \vec{\nabla} \times \vec{B})(\vec{x})(\vec{B} \cdot \vec{\nabla} \times \vec{B})(\vec{x}+\vec{r})\rangle}{\left\langle|\vec{B}(\vec{x})|^{2}\right\rangle^{2}}+O(g(R)) .
\end{aligned}
$$

Notice that to estimate the numerator appearing on the righthand side of Eq. (4.16), Eq. (4.14) can be used together with the considerations reported in Appendix A.

\section{PHENOMENOLOGICAL IMPLICATIONS}

Having set all the formalism for computing baryonnumber fluctuations, we now come to the physical consequences. As we argued in Sec. III B, these fluctuations survive after the electroweak phase transition only if it is strongly first order; we will assume that this is the case. We will argue in Sec. VI A that strong enough magnetic fields make an EW phase transition strongly first order even in the case of the minimal standard model. Otherwise, some extension of it can be considered.

An essential quantity entering all expressions for baryonnumber fluctuations is the ratio between the perturbative and nonperturbative rate of the right-electron chirality flip. Fluctuations are larger for $\Gamma>\Gamma_{\mathcal{H}}$. In Secs. V A and V B we will assume that this is indeed the case, and analyze this assumption in detail in Sec. V C.

As a preliminary warmup let us estimate the amplitude of baryon-number fluctuations at the magnetic diffusivity scale for a flat spectrum of magnetic fields $(\epsilon \ll 1)$. If the energy sitting in the background magnetic field is comparable with the energy density of the photons, $\left\langle\overrightarrow{\mathcal{H}}_{Y}^{2}\right\rangle \sim T_{c}^{4}$, then for the 

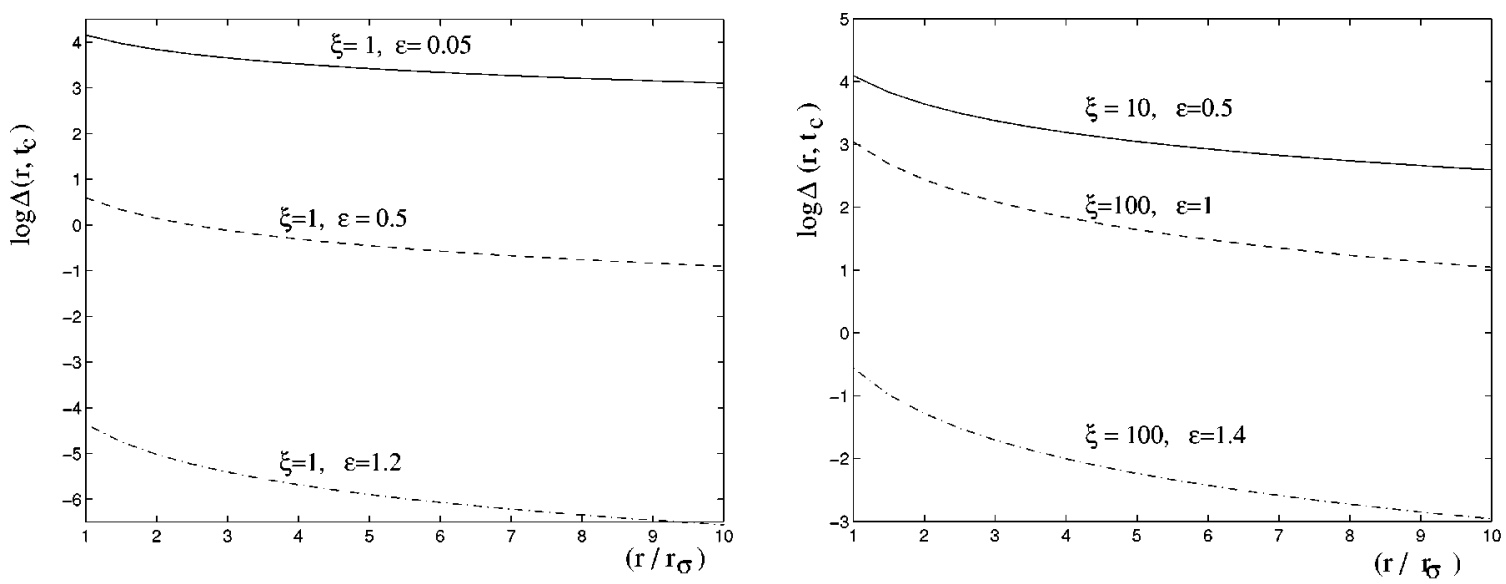

FIG. 1. We plot the level of fluctuations given in Eq. (4.15) for different values of amplitudes $(\xi)$ and slopes $(\epsilon)$ of the hypermagnetic energy spectrum for scales slightly larger than the diffusivity scale, which is $10^{-9} \times L_{\mathrm{EW}}\left[L_{\mathrm{EW}}\right.$ is the EW horizon, see also Eq. (3.8)]. These scales will not be washed out by the finite value of the conductivity, and we can immediately see that for a sufficiently blue spectrum $(\epsilon \ll 1) \Delta \sim 10^{3}-10^{4}$ as suggested by the estimate of Eq. (5.1). Looking at the plots from top to bottom, we see that $\Delta$ decreases for increasingly violet spectra $(\epsilon \gtrsim 1)$ and for fixed amplitude $(\xi)$ of the hypermagnetic energy spectrum. We notice that the results illustrated in this plot depend very weakly upon the value of the hyperconductivity. In particular, in the present plots, we assumed $\sigma_{c} / T_{c} \simeq 70$ as fiducial value for the hyperconductivity at the EW scale.

smallest possible scale $r \sim 1 / k_{\sigma} \sim 10^{-9}$ (EW horizon $\simeq 3 \mathrm{~cm}$ ) we get, from Eq. (4.15),

$$
\Delta\left(r_{\sigma}, t_{c}\right) \sim \frac{\alpha^{\prime}}{N_{\text {eff }}} \sqrt{\frac{M_{0}}{\sigma_{c}}} \sim 10^{3} .
$$

This estimate is certainly quite large and it is unlikely to be correct, since for such huge fluctuation the backreaction of the created fermions on the magnetic fields and on the dynamics of the electroweak phase transition (which we ignored) must be taken into account. Nevertheless, it shows that considerable inhomogeneities in the baryonic number are possible on small scales. The estimate (5.1) considerably exceeds the measure of the baryon asymmetry of the universe $n_{B} / s \sim 10^{-10}$, thus small size matter-antimatter are possible at the EW scale. At the same time, for even larger scales (possibly relevant for structure formation), the fluctuations of Eq. (4.15) are quite minute (since their amplitude decreases with the distance as $\left.1 / r^{1+\epsilon}\right)$ and may be safely neglected.

The above estimate suggests that a quite natural outcome of the presence of stochastic background of the primordial hypercharge field may be a rather inhomogeneous distribution of matter and antimatter domains for scales inside the EW horizon. The fluctuations estimated in Eq. (4.15) are also illustrated in Fig. 1 where the level of fluctuation is plotted for different slopes and amplitudes of the hypermagnetic energy spectrum. We clearly see that by tuning $\xi$ (i.e., by tuning the amplitude of the hypermagnetic energy spectrum) the level of induced matter-antimatter fluctuations can be as large as suggested by the estimate of Eq. (5.1).

We will now discuss the relevance of the generated fluctuations for the BBN. In fact sizable matter-antimatter fluctuations can provide a new type of initial conditions for nonhomogeneous BBN. From a more conservative point of view, we can instead assume that the BBN was essentially homogeneous; then our considerations provide a new bound on primordial magnetic fields present at the EW epoch. For completeness we will compare the bounds arising from the occurrence of matter-antimatter domains for scales of the order of the neutron diffusion distance with the bounds for magnetic fields that are at present coherent over much larger scales.

\section{A. BBN and matter-antimatter fluctuations}

The success of the homogeneous and isotropic nucleosynthesis may impose strong constraints upon the baryonnumber fluctuations possibly produced prior to the formation of the light nuclei. Broadly speaking, the predictions of homogeneous BBN for the primordial abundances of the light elements are compatible with the observations only if the baryon-to-photon ratio lies in a quite narrow range around $n_{B} / n_{\gamma}=3 \times 10^{-10}-10^{-9}$ [39].

Generally, if $\Delta\left(r, t_{c}\right)>n_{B} / s$ for some length scale $r$, we have to conclude that matter-antimatter domains will be formed. If, on the other hand, $\Delta\left(r, t_{c}\right)<n_{B} / s$ at all scales, only positive-definite fluctuations in baryonic density are produced.

Upper and lower limits on the scales over which a perturbation in baryon number can affect nucleosynthesis through neutron-proton segregation are determined by the comoving diffusion lengths of neutrons and protons at the beginning of nucleosynthesis. At high temperatures the diffusion lengths of neutrons and protons are almost the same, since neutronproton equilibrium is guaranteed by weak interactions. After weak interactions have fallen out of equilibrium, nucleons retain their identity as neutrons and protons, and diffusive segregation can occur. Coulomb scattering between protons and electrons (or positrons) give a cross section roughly equal to the Thompson cross section. Since neutrons have a magnetic moment they scatter electrons with a cross section of $8 \times 10^{-31} \mathrm{~cm}^{2}$. Neutrons scatter also nucleons and the scattering cross section in terms of the triplet and singlet scattering lengths is roughly $2.3 \times 10^{-23} \mathrm{~cm}^{2}$. Once the cross sections of the processes involved are known, the diffusion 
scale is simply given by $L\left(\tau_{U}\right) \sim \sqrt{6 \tau_{U} D\left(\tau_{U}\right)}$, where $D\left(\tau_{U}\right)$ is the diffusion coefficient (usually related to the mobility through the Einstein coefficient [40]) at any given time $\tau_{U}$ $=M_{0} / T^{2}$.

At the onset of nucleosynthesis $\left(T_{\mathrm{ns}} \simeq 0.2 m_{e}\right.$, where $m_{e}$ is the electron mass) the comoving diffusion scale turns out to be [41-44] $3 \times 10^{5} \mathrm{~cm}$. The neutron diffusion length blueshifted at the time of the electroweak phase transition is

$$
L_{\text {diff }}\left(T_{c}\right)=0.3 \mathrm{~cm} \text { for } T_{c}=100 \mathrm{GeV} .
$$

If $\Delta\left(L_{\text {diff }}, t_{c}\right)>n_{B} / s$ matter-antimatter domains will not be erased by the nucleosynthesis time and, at the same time, fluctuations occurring over scales smaller than $L_{\text {diff }}\left(t_{c}\right)$ at the electroweak epoch are likely to be dissipated [41,42].

Taking again the flat spectrum for magnetic fields and assuming that their energy is $\sim T^{4}$, we thus obtain for the baryon-number fluctuations at that scale $\delta\left(n_{B} / s\right) \sim 10^{-5}$ $\gg 10^{-10}$. If magnetic fields are large enough (with sufficiently flat spectra), domains of matter and antimatter may exist at scales five orders of magnitude larger than the neutron diffusion length. ${ }^{2}$ To our best knowledge, there have been no studies of nonhomogeneous BBN with this type of initial conditions. Of course, there were a lot of investigations of non-homogeneous nucleosynthesis, motivated by first-order quark-hadron phase transition [45]. In particular, baryon-number fluctuations with spectral amplitudes growing in frequency (and then decaying at large length scales) were recently addressed [43], with the result that these fluctuations are allowed, provided they occur at scales smaller than the neutron diffusion length. However, Refs. [41-44] essentially considered positive-definite baryon-number fluctuations, rather than with matter-antimatter domains. These results were also used in order to constrain the possible baryon-number fluctuations arising in the context of topological defect models of baryogenesis [46]. It would be very interesting to see whether matter-antimatter domains may change BBN bounds on the baryon-to-photon ratio by changing the related predictions of the light element abundances. This possible analysis will not be attempted here.

We can instead adopt a more conservative attitude and require that no matter-antimatter domains larger than the nucleon diffusion scale exist at the onset of nucleosynthesis. This will give some constraints on the primordial hypercharge magnetic fields. In order not to have matter-antimatter domains affecting BBN, we therefore demand

$$
\Delta\left(L_{\text {diff }}, t_{c}\right)<\frac{n_{B}}{s} .
$$

From Eq. (4.15), imposing the bound (5.3), we can translate the constraints coming from homogeneity of BBN into an exclusion plot in terms of the only two parameters ( $\epsilon$ and $\xi)$ characterizing our stochastic hypermagnetic background:

\footnotetext{
${ }^{2}$ Note that the energy fluctuations of the electroweak horizon scale are always sufficiently small (i.e., $\delta \rho_{p} / \rho_{p} \ll 1$ for $r \sim L_{\mathrm{EW}}$ ) so that black-hole formation is not expected.
}

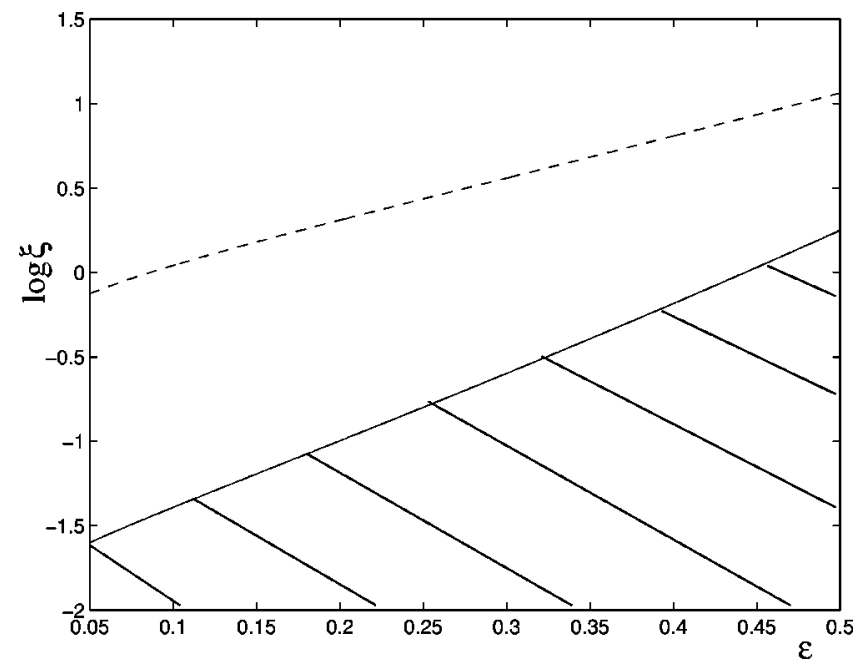

FIG. 2. We plot the constraint on the stochastic background of magnetic hypercharge field derived in Eq. (5.4) by requiring the homogeneity in the baryon-number fluctuations at the neutron diffusion length. We have chosen $\Omega_{B} h_{100}^{2}=0.01$ and $\Omega_{0} h_{100}^{2} \sim 1$. We notice that by changing $h_{100}$ in the range $0.4<h_{100}<1$ the quantitative change in the bound is negligible. We also took $\sigma_{c} / T_{c} \sim 70$ as fiducial value. The variation of $\sigma_{c}$ in a plausible range does not alter the features of the present plot. The shaded region in the parameter space corresponds to matter-antimatter fluctuations that will be erased by the nucleosynthesis time, whereas in order to have sizable matter-antimatter domains at the onset of $\mathrm{BBN}$ we have to go above the line. In the dashed line we report the critical energy density bound given in Eqs. (5.5) and (5.6).

$$
\begin{aligned}
\log _{10} \xi< & \left(-6.262+\log _{10} \frac{\sigma_{c}}{T_{c}}+\frac{1}{2} \log _{10} \epsilon+(14.88) \epsilon\right. \\
& \left.+\log _{10}\left[\Omega_{B} h_{100}^{2}\right]\right) /(4-\epsilon) .
\end{aligned}
$$

This condition is reported in Fig. 2 where, for the validity of our approximations, we considered the case where $\epsilon \geq 0.05$ [for $\epsilon \lesssim 0.05$ and scales $L_{\text {diff }} / r_{\sigma} \gtrsim 10^{9}$ the corrections appearing in Eq. (4.15) are not under control]. We plotted our bounds in the case $0.05 \leq \epsilon \leq 1$. There is a second constraint which one might want to impose on our background, namely, the one coming from the critical energy density:

$$
\begin{gathered}
\rho_{H}\left(t_{c}\right)<\rho_{\gamma}\left(t_{c}\right), \quad \rho_{H}\left(t_{c}\right)=\frac{1}{2}\left\langle|\vec{H}(\vec{x})|^{2}\right\rangle, \\
\rho_{\gamma}\left(t_{c}\right)=\frac{\pi^{2}}{30} N_{\mathrm{eff}} T_{c}^{4}
\end{gathered}
$$

[where $\left\langle|\vec{H}(\bar{x})|^{2}\right\rangle$ is given by Eq. (4.5)]. Using now Eq. (4.6) we can convert Eq. (5.5) into a further (but milder) constraint on our parameter space

$$
\begin{aligned}
\log _{10} \xi< & \left(\log _{10} \frac{\pi N_{\text {eff }}}{120}+\frac{\epsilon}{2}\left[\log _{10} 2-\log _{10} \frac{\sigma_{c}}{M_{0}}\right]\right. \\
& \left.+\log _{10} \epsilon\right) /(4-\epsilon)
\end{aligned}
$$

This bound is also reported in Fig. 2 (dashed line). Sizable matter-antimatter domains are produced when the spectrum 

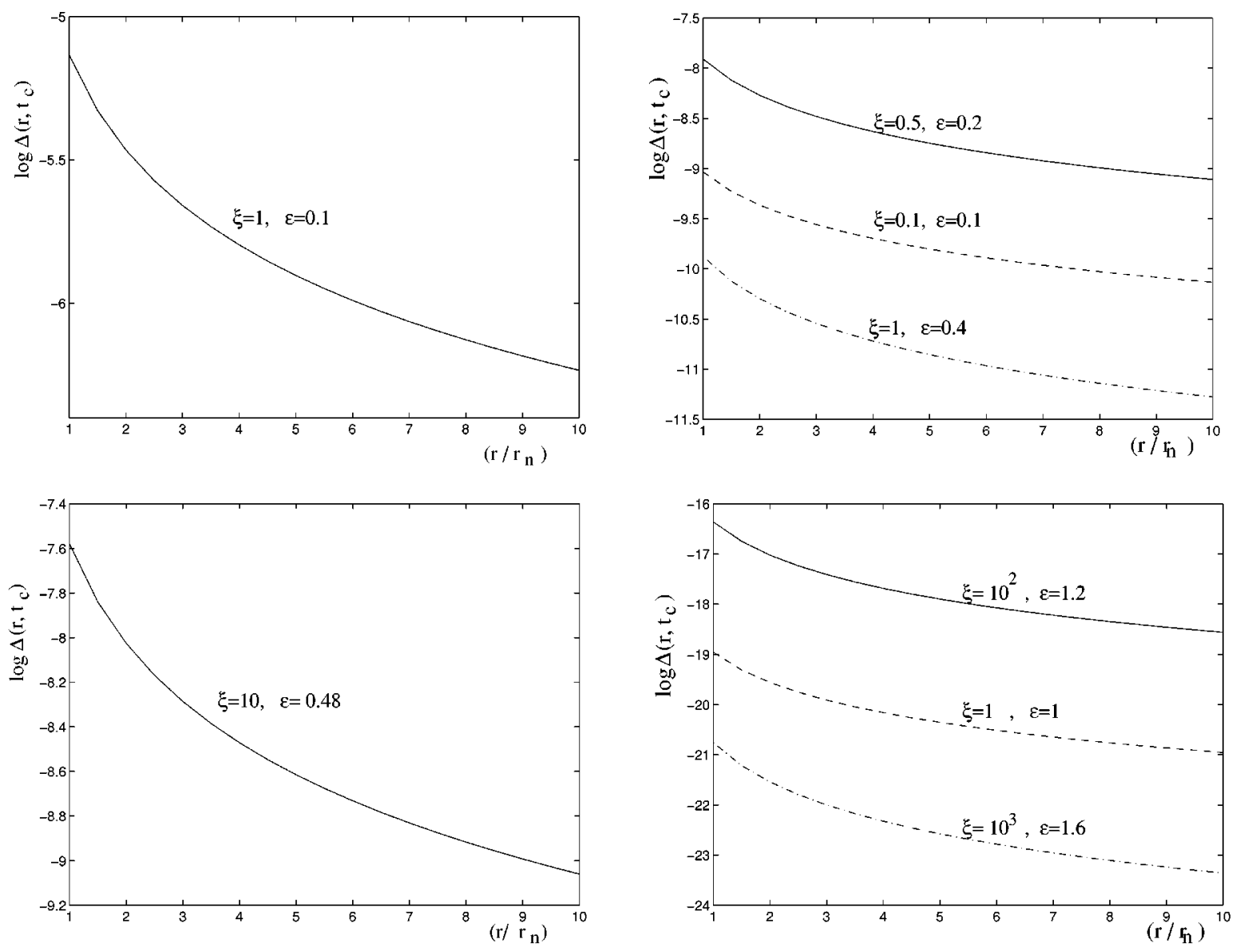

FIG. 3. We plot the expected level of fluctuations given in Eq. (4.15) for scales of the order of (and larger than) the neutron diffusion distance given in Eq. (5.2). In the plot $r_{n}=L_{\text {diff }}$. In this case we always took $\sigma_{c} / T_{c} \sim 70$. We can notice that for flat enough spectra a very interesting level of fluctuations is allowed. For violet spectra the fluctuations are certainly suppressed. The same trend exists for even larger scales.

is sufficiently flat. This feature can also be deduced from the level of fluctuations for scales of the order of the neutron diffusion distance, which we plot in Fig. 3. We see that it is quite possible to get $\Delta \gg 10^{-10}$ around the neutron diffusion distance.

We want to notice that an artificial way of relaxing our exclusion plot (5.4) could be (trivially) to enhance the level of the baryon asymmetry by enhancing $\Omega_{B} h_{100}^{2}$ (up to values of the order of 0.1-1). This phenomenon turns out to be similar to the one discussed in [47], where it was argued that baryon-number fluctuations with blue frequency spectra might offer an interesting mechanism for accounting for large amounts of baryonic dark matter. This is purely an analogy, since the problem we are discussing (as we stressed) is not the origin of the baryon asymmetry, but the possible bounds on the hypercharge fields. Therefore the value of $\Omega_{B} h_{100}^{2}$ is an external parameter for us, but not a computable number (see also Sec. VI).

A comment is now in order concerning the phenomenological estimates we made in this subsection. The system of Eqs. (2.22) and (3.1) was solved in the approximation of local thermal equilibrium and the possible backreaction effects were ignored. Numerical solutions of this nonlinear system of partial differential equations are required if the level of induced fluctuations gets too large (i.e., $\Delta \sim 1$ ). We will not discuss here how to address this complicated nu- merical problem, but we will come back to it in Sec. VI.

\section{B. Comparison with other bounds on primordial magnetic fields}

In this section we are going to compare the BBN bound reported in Eq. (5.4) with other possible bounds, which could be applied on primordial magnetic fields.

It is well known that there are direct bounds on primordial magnetic fields at the nucleosynthesis epoch [20]. Moreover, quite recently, two constraints on magnetic field intensities were derived using, respectively, the anisotropies in the microwave sky [21] and the Faraday rotation correlations [24]. These last two bounds apply of course to the case of fields that are, today, coherent over length scales much broader than the (present) nucleosynthesis scale. In order to see how stringent our bound is, we should compare it with the ones already available and coming from larger scales. In this sense our aim is to show that our bounds are clearly more stringent for small-scale fields but cannot compete (at even larger scales) with the ones coming from the CMBR anisotropies and from the Faraday rotation measurements.

Since the CMBR is isotropic to a very high degree of accuracy, its small anisotropies can constrain the intensity of a constant magnetic field (coherent over the present horizon size [21]), which could modify the evolution equations of the 


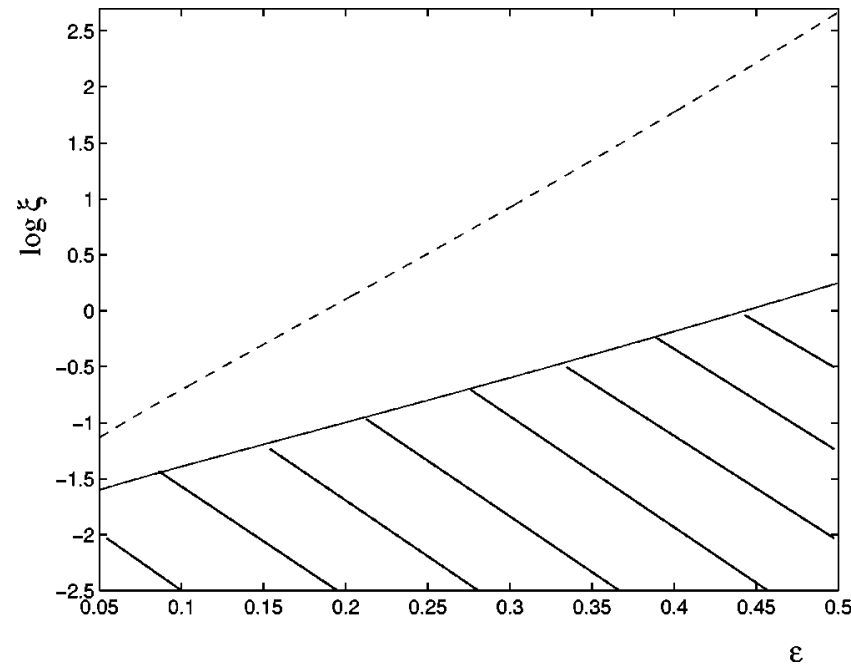

FIG. 4. We plot our bound (lower curve) obtained in Eq. (5.4) and the bound derived in $[21,22]$ (upper curve) from the CMBR anisotropies in the case of a magnetic field coherent over the horizon size today. For values of $\epsilon$ and $\xi$ above the shaded region the level of fluctuations exceeds the bound (5.3). Again the variation of $\Omega_{B} h_{100}^{2}$ (possibly between 0.1 and 0.01 ) changes the plot by only a few percent. In this plot we took $h_{100}=0.6$.

matter sources by introducing a slightly anisotropic pressure [22]. The calculation of the CMBR anisotropies can be carried out also in the case of slightly skew stresses, whose numerical weight depends upon the magnetic field intensity. By comparing the final result with the level of anisotropies detected by COBE, it is possible to compute how big the magnetic field intensity should be not to conflict with observed anisotropies in the microwave sky. At the present time, the constraint is $\left|\vec{H}_{0}\left(t_{0}\right)\right|<6.8 \times 10^{-9}\left(\Omega_{0} h_{100}^{2}\right)^{1 / 2} \mathrm{G}$ over a length scale $L_{0}\left(t_{0}\right) \simeq 9.25 \times 10^{27} h_{100}^{-1} \mathrm{~cm}$. The authors of Refs. [21, 22] gave the bound in terms of $h_{50}$ (the present uncertainty in the Hubble parameter in units of $50 \mathrm{~km} / \mathrm{Mpc}$ $\mathrm{sec})$. For consistency with our notation and in order to make the comparison with other bounds easier, use instead $h_{100}$ $=h_{50} / 2$ taking, as usual, $0.4<h_{100}<1$. By blueshifting the bound of [21] up to $T_{c}$ we get $\left|\vec{H}_{0}\left(t_{c}\right)\right|<1.12$ $\times 10^{22}\left(\Omega_{0} h_{100}^{2}\right)^{1 / 2} \mathrm{G}$ at a scale $L_{0}\left(t_{c}\right) \simeq 7.19 \times 10^{12} h_{100}^{-1} \mathrm{~cm}$, where we assumed that the magnetic field scales as $1 / a^{2}(\tau)$, as it is plausible to demand for length scales larger than the magnetic diffusivity scale. We also took into account the evolution in the effective number of degrees of freedom in the plasma, which is $N_{\text {eff }}\left(t_{c}\right) \sim 106.75$ and $N_{\text {eff }}\left(t_{\text {dec }}\right) \sim 3.90$. The bound on $H_{0}\left(t_{c}\right)$ turns into a bound on $B\left(t_{c}\right)$ $=\sqrt{\left\langle H^{2}\right\rangle} / T_{c}^{2}$ (recall that $\left.1 \mathrm{G}=1.95 \times 10^{-20} \mathrm{GeV}^{2}\right)$.

From Eq. (4.6) [taking $r \sim L_{0}\left(t_{c}\right), T \sim T_{c}$ ] it actually results that the parameter space of our model has to satisfy the following requirement:

$$
\begin{aligned}
\ln \xi< & \left(-2.30+\frac{1}{2} \ln \left[\Omega_{0} h_{100}^{2}\right]+\frac{1}{2} \ln \epsilon+[14.13\right. \\
& \left.\left.-\frac{1}{2} \ln h_{100}\right] \epsilon\right) /\left(2-\frac{\epsilon}{2}\right)
\end{aligned}
$$

For comparison this constraint is reported in Fig. 4, together with the bound of Fig. 2. Since the region defined by Eq. (5.4) is always below the curve of Eq. (5.7), we conclude that the bound imposed by the homogeneous BBN is more constraining than the one reported in $[21,22]$ for $\epsilon>0.05$. Clearly, for some very small slopes, the COBE bound will become better, but we cannot compute this critical value of $\epsilon$ since our approximation breaks down before it. In [24] the polarized emission of a few hundred galaxies was reviewed and another bound on the present intensity of large-scale magnetic fields was reported. A large-scale field should produce an additional shift in the polarization plane of the incoming radiation according to the Faraday effect. In fact the polarization plane of the synchrotron radiation gets shifted, in the background of a magnetic field, by an amount that is directly proportional to the integral of the magnetic field times the electron density along the line of sight. By subtracting, from the total angular shift, the one produced by each galactic field, it is possible to constrain the intensity of any field coherent over scales larger than the galactic one. The only uncertainty with this procedure is that the measurements assume that the magnetic fields of the Milky Way and of the other galaxies are known to a very high degree of accuracy, since they have to be subtracted from each estimate of the Faraday rotation. The constraint obtained with this method turns out to be $\left|\vec{H}_{1}\left(t_{0}\right)\right|<1 \times[(2.6$ $\left.\left.\times 10^{-7} \mathrm{~cm}^{-3}\right) / \bar{n}_{B}\right] h_{100} \times 10^{-9} \mathrm{G}$, for fields now coherent over scales $L_{1}\left(t_{0}\right) \simeq(10-50) h_{100}^{-1} \mathrm{Mpc}$. Assuming that the mean (present) baryon density is $\bar{n}_{B} \sim 1.13$ $\times 10^{-5}\left(\Omega_{B} h_{100}^{2}\right) \mathrm{cm}^{-3}$, the (blueshifted) field intensity and its coherence length will be, respectively, $\left|\vec{H}_{1}\left(t_{c}\right)\right|<3.79$ $\times 10^{19}\left(\Omega_{B} h_{100}^{2}\right)^{-1} h_{100} \mathrm{G}$ and $L_{1}\left(t_{c}\right) \simeq 6 \times 10^{10} h_{100}^{-1} \mathrm{~cm}$ where we took $L_{0}\left(t_{0}\right) \simeq 25 \mathrm{Mpc}$. By translating this bound in the $(\xi, \epsilon)$ plane, we obtain the following relation:

$$
\begin{aligned}
\log _{10} \xi< & \left(-4.77+\frac{1}{2} \log _{10} \epsilon-\frac{1}{2} \log _{10} \Omega_{B} h_{100}^{2}+[13.09\right. \\
& \left.\left.-\frac{1}{2} \log _{10} h_{100}\right] \epsilon\right) /\left(2-\frac{\epsilon}{2}\right)
\end{aligned}
$$

This constraint is illustrated in Fig. 5, where we see that our curve is always below the curve reported in Eq. (5.8). Our constraint is again more stringent than the one of Eq. (5.8) for $\epsilon>0.05$. The bounds on ordinary magnetic fields at the nucleosynthesis epoch also apply in our case. In order not to affect the universe expansion at nucleosynthesis it should hold (see, for instance, Kernan et al. in Ref. [20]) that $\rho_{H}$ $<0.27 \rho_{\nu}$ [where $\rho_{H}$ is the magnetic energy density defined in Eq. (5.5)] and $\rho_{\nu}$ is the energy density contributed by the standard three light neutrinos for $T \ll 1 \mathrm{MeV}$. Therefore in terms of $\xi$ and $\epsilon$ this bound reads:

$$
\log _{10} \xi<\frac{\left(11.30-\frac{1}{2} \log _{10} \sigma_{c} / T_{c}\right) \epsilon+\log _{10} \epsilon-0.2}{4-\epsilon} .
$$

This bound is reported in Fig. 6 and is compared with our bound. We see that the bound coming from the absence of matter-antimatter domains at the nucleosynthesis epoch is more constraining for (by two orders of magnitude for logarithmic energy spectra with $\epsilon \ll 1)$. Note also that, according 


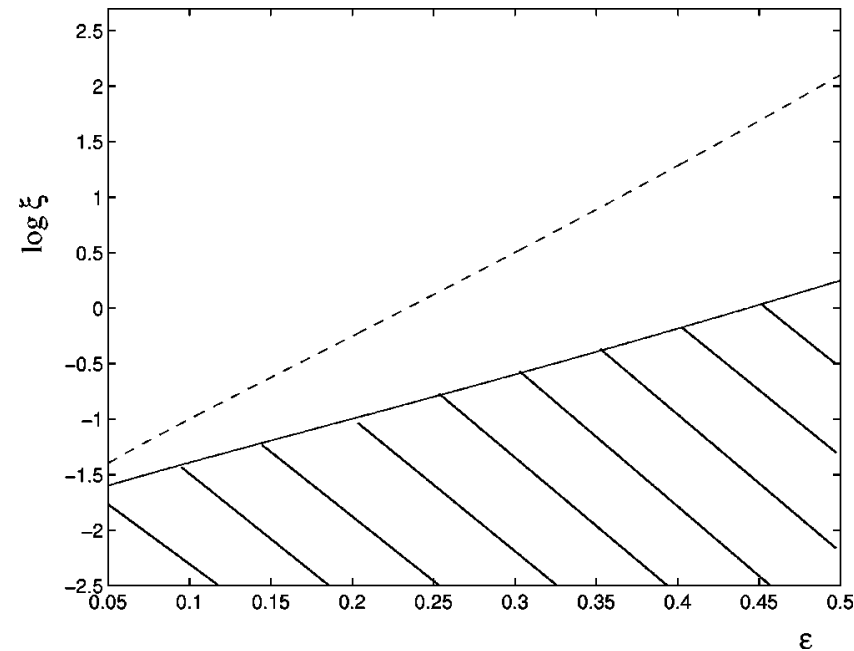

FIG. 5. We compare the bounds of Eqs. (5.4) and (5.8). The most constraining bound is the one given by the lower curve given by Eq. (5.4). The upper curve derived in the context of Faraday rotation measurements is more constraining than the one obtained from the isotropy of the CMBR. The numerical value of the parameters for which this plot is obtained is the same as for Figs. 2 and 4.

to [48], the bound (5.9) may in fact be absent, because there are other mechanisms, besides magnetic diffusivity, that can dilute the magnetic fields before the BBN. Then our bound remains the only one that can be applied to the small-scale magnetic fields.

Another interesting numerical value of the magnetic field, which might be compared with our considerations is $\left|\vec{H}_{3}\left(t_{\text {dec }}\right)\right| \simeq 10^{-3} \mathrm{G}$ coherent over a scale $L_{3}\left(t_{\mathrm{dec}}\right) \simeq 1.690$ $\times 10^{23}\left(\Omega_{0} h_{100}^{2}\right)^{-1 / 2} \mathrm{~cm}$, which is the size of the horizon at the decoupling. If this field would be present at the decoupling time (when the temperature was roughly $T_{\text {dec }}=0.258 \mathrm{eV}$ ) it might also rotate the polarization plane of the CMBR provided the CMBR is weakly polarized [23]. At

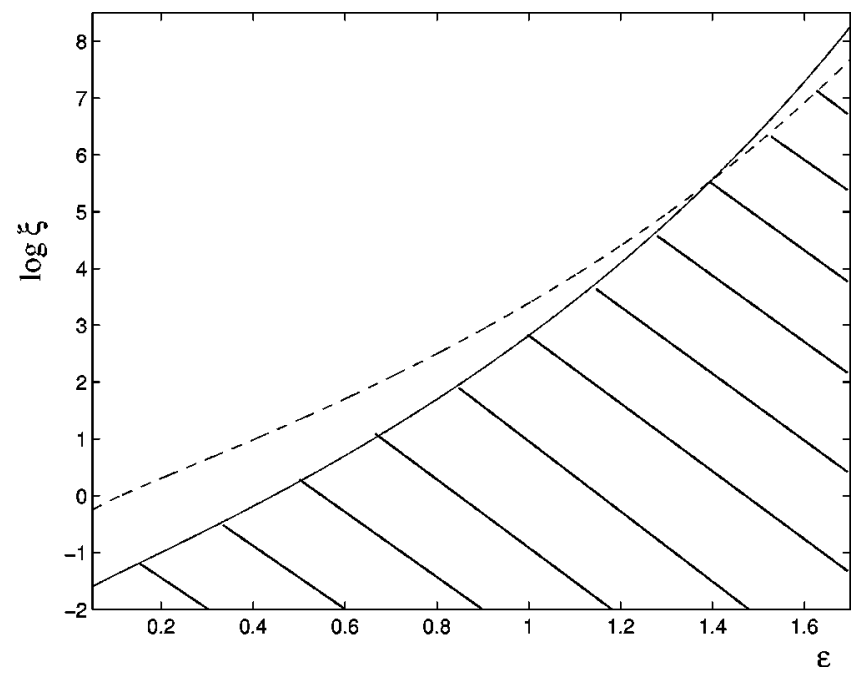

FIG. 6. We compare the direct bound on primordial magnetic fields at the nucleosynthesis epoch derived in Eq. (5.9) with the bound derived in Eq. (5.4), which applies to primordial magnetic fields. We see that our requirement is again more constraining than the one given in Refs. [20] for blue spectra, whereas for $\epsilon \geq 1.4$ the bound given by Eq. (5.9) is better.

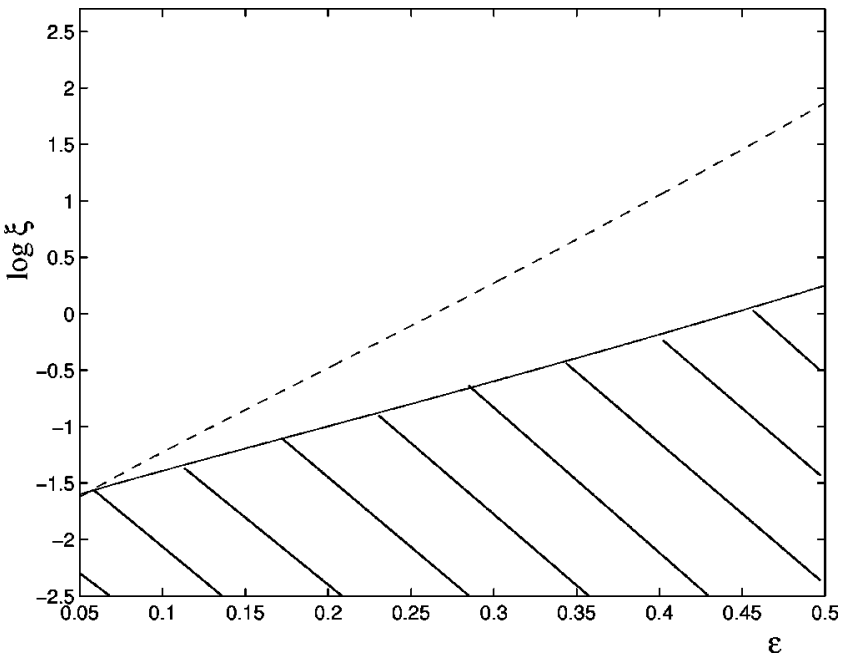

FIG. 7. We plot the constraint derived on the basis of BBN considerations [Eq. (5.4)] together with the requirement derived in Eq. (5.10). The dashed line corresponds to a magnetic field of EW origin strong enough to rotate the polarization plane of the CMBR under the assumption that the CMBR is polarized. We can see that if such a field has an electroweak origin, then also sizable matterantimatter fluctuations will be present for scales of the order of the neutron diffusion distance.

the scale of the electroweak phase transition the blueshifted field and the corresponding correlation length read, respectively, $\quad\left|\vec{H}_{3}\left(t_{c}\right)\right| \simeq 1.36 \times 10^{21} \mathrm{G} \quad$ and $\quad L_{3}\left(t_{c}\right) \simeq 1.447$ $\times 10^{11}\left(\Omega_{0} h_{100}^{2}\right)^{-1 / 2} \mathrm{~cm}$ and this imposes for our parameters the following requirement:

$$
\begin{aligned}
\log _{10} \xi \gtrsim & \left(-3.22+\frac{1}{2} \log _{10} \epsilon+13.28 \epsilon\right. \\
& \left.-\frac{\epsilon}{4} \log _{10}\left[\Omega_{0} h_{100}^{2}\right]\right) /\left(2-\frac{\epsilon}{2}\right) .
\end{aligned}
$$

It is of some interest to notice from Fig. 7 that the region defined by Eq. (5.10) falls in the forbidden area of Fig. 2. This means that the BBN bound of Eq. (5.4) excludes the possibility that a primordial magnetic field of EW origin is strong enough to rotate the polarization plane of the CMBR. On the other hand, if nonstandard initial conditions for the inhomogeneous BBN scenario (i.e., matter-antimatter domains) would be allowed, this conclusion might be relaxed and the existence of such an intense field at the decoupling epoch might be accommodated. It is anyway amusing that in our present discussion the existence of a magnetic field at the decoupling epoch might imply the presence of small-scale antimatter domains at the onset of BBN.

\section{The rate of right-electron chirality flip}

In Sec. III we pointed out how important, in our context, the interplay between the "perturbative" rate given by the right-electron chirality flip processes and the "nonperturbative" one coming from the anomaly is. For the MSM the perturbative rate of chirality flip has been computed in [31] and is determined by the right-electron Yukawa coupling. If MSM is a correct theory, then $\Gamma>\Gamma_{\mathcal{H}}$ only for extremely 
small magnetic fields, $H^{2} / T^{4}<\frac{22}{783}\left(\sigma_{0} / \alpha^{\prime}\right)\left(T_{R} / M_{0}\right)$ $\simeq 10^{-11}$. So weak hypermagnetic fields do not produce any interesting fluctuations. For larger magnetic fields the approximation outlined in Eq. (3.4) must be used. The amplification factor that appears in Eq. (3.9) can be extracted from [31]

$$
\begin{aligned}
\int_{0}^{t_{c}} d t \Gamma \simeq & \frac{\Gamma M_{0}}{T_{c}^{2}} \\
\simeq & 350\left(\frac{100 \mathrm{GeV}}{T_{c}}\right)\left[\left(-1.1+2.4 x_{H}\right)+1.0\right. \\
& \left.+h_{t}^{2}\left(0.6-0.09 x_{H}\right)\right]
\end{aligned}
$$

where $x_{H}=\lim _{T \rightarrow \infty} m_{H}(T) / T$ is the high-temperature limit of the Higgs boson thermal mass and $h_{t}$ is the top-quark Yukawa coupling. Taking $x_{H} \simeq 0.6$ (for which the scattering contribution to the rate is always dominant with respect to the decay contribution), we find that the integrated rate is $655 \times\left(100 \mathrm{GeV} / T_{c}\right)$. With the use of this number the analysis of Sec. V can be redone with the result that no interesting baryon-number fluctuations can be produced from stochastic hypermagnetic background. So, for MSM, one hardly expects any cosmological consequences coming from the background of the type (4.4) (for other types of primordial hypermagnetic fields, considered below in Sec. VI B, the conclusion is different).

However, in the extensions of the standard model, the rate $\Gamma$ can be naturally larger than in the MSM. For example, in the context of the minimal supersymmetric standard model (MSSM) the right-electron Yukawa coupling is enhanced by a factor $1 / \cos \beta(\tan \beta$ gives the ratio of the expectation values of the two doublets), so that $T_{R}$ can be larger by a factor $10^{3}$ for experimentally allowed values of $\tan \beta \sim 50$. Moreover the right-electron number is now shared between electrons and selectrons, and it is necessary to consider also processes that change the selectron number.

The question we now want to address is more phenomenological. Namely, we want to see how large the perturbative chirality flip rate $\Gamma$ should be in order to produce sizable matter-antimatter fluctuations, which could influence the BBN. For this purpose, we just require that $\Gamma>\Gamma_{\mathcal{H}}$, with $\Gamma_{\mathcal{H}}$ taken from Eqs. (3.9) and (4.6), and use the minimal amplitude of the hypermagnetic field obtainable from the bound (5.4), which can produce sizable matter-antimatter fluctuations. This gives $\Gamma / T_{c}>10^{-9}$, which corresponds to the right-electron perturbative freezing temperature $T_{R}$ $=10^{5} \mathrm{TeV}$. As we discussed above, these values of the temperature are perfectly possible, say, in the MSSM. If the actual value of the freezing temperature is smaller than $10^{5} \mathrm{TeV}$, the stochastic hypercharge background of type (4.4) produces baryon-number fluctuations too small to affect BBN. It is then interesting that a quite energetic stochastic hypermagnetic background can be accommodated in the MSM without any (potentially dangerous) implications. The energy density stored in this background can then be able to influence the dynamics of the EW phase transition without conflicting with any bound derived from BBN. This will be one of the subjects of the following section.

\section{EW PHASE TRANSITION AND BARYOGENESIS}

The aim of this section is the discussion of the influence of the hypercharge magnetic field on the electroweak baryogenesis (for reviews, see [49]). First, we will consider the EW phase transition in the presence of the hypermagnetic field. Then, we will show that the occurrence of some specific hypermagnetic configurations in the EW plasma could be responsible for the baryon asymmetry of the universe (BAU).

\section{A. EW phase transition}

The hypercharge magnetic field, present at high temperature, can influence the dynamics of the phase transition. The physical picture of this phenomenon is exactly the same as the macroscopic description of superconductors in the presence of an external magnetic field. The normalsuperconducting phase transition, being of second order in the absence of magnetic fields, becomes of first order if a magnetic field is present. The reason for this is the Meissner effect, i.e., the fact that the magnetic field cannot propagate inside a superconducting cavity, and, therefore, creates an extra pressure acting on the normal-superconducting boundary [50]. Our consideration below explores this simple picture.

Consider the plain domain wall that separates broken and symmetric phase at some temperature $T$, in the presence of a uniform hypercharge magnetic field $\mathcal{Y}_{j}$. Far from the domain wall, in the symmetric phase, the non-Abelian SU(2) field strength $\left(\mathcal{W}_{j}^{3}\right)$ is equal to zero, because of a nonperturbative mass gap generation. Inside the broken phase, the massive $Z_{j}$ combination of $\mathcal{Y}_{j}$ and $\mathcal{W}_{j}^{3}$,

$$
Z_{j}=\cos \theta_{W} \mathcal{W}_{j}^{3}-\sin \theta_{W} \mathcal{Y}_{j},
$$

must be equal to zero, while the massless combination, corresponding to photon $A_{j}^{\mathrm{em}}$, survives. The matching of the fields on the boundary gives $A_{j}^{\mathrm{em}}=\mathcal{Y}_{j} \cos \theta_{W}$. Thus an extra pressure $\frac{1}{2}\left|\overrightarrow{\mathcal{H}}_{Y}\right|^{2} \sin ^{2} \theta_{W}$ acts on the domain wall from the symmetric side. At the critical temperature it must be compensated by the vacuum pressure of the scalar field. If we neglect loop corrections associated with the presence of magnetic fields, then the condition that determines the critical temperature is

$$
\frac{1}{2}\left|\overrightarrow{\mathcal{H}}_{Y}\right|^{2} \sin ^{2} \theta_{W}=V\left(0, T_{c}\right)-V\left(\varphi_{\min }, T_{c}\right),
$$

where $V(\varphi, T)$ is the effective potential in the absence of magnetic field, $\varphi_{\min }$ is the location of the minimum of the potential at temperature $T$.

The above consideration was dealing with the uniform magnetic fields. Clearly, it remains valid when the typical distance scale of inhomogeneities of magnetic field is larger than the typical bubble size. This is the case for bubbles smaller than the magnetic diffusion scale, and, in particular, at the onset of the bubble nucleation. Thus the estimate of the critical temperature coming from Eq. (6.2) is applicable. For bubbles larger than the diffusivity scale, the presence of 


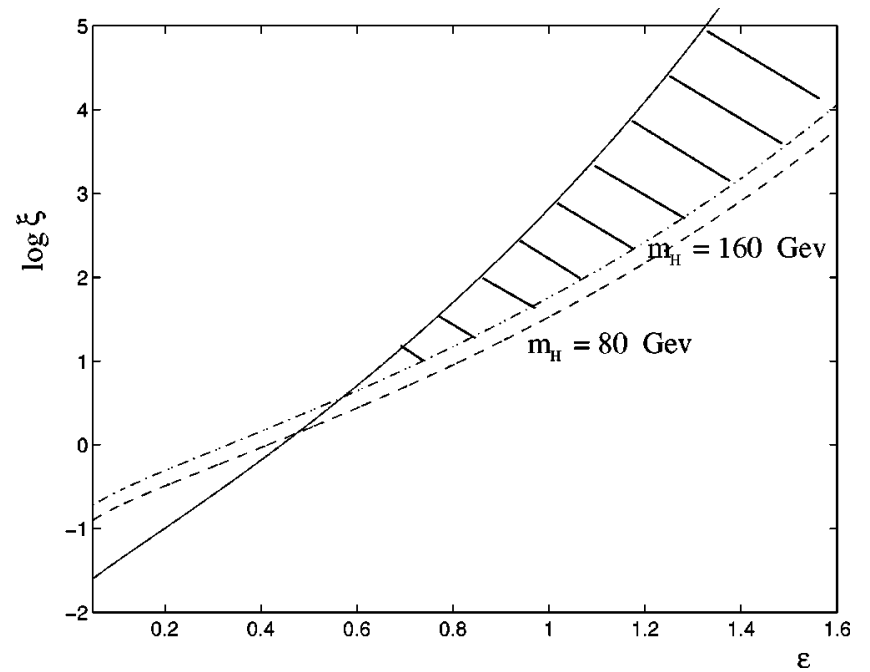

FIG. 8. We plot the requirement obtained in Eq. (6.2) in the case of a stochastic hypermagnetic background. The dashed line refers to the case of $m_{H}=80 \mathrm{GeV}$, whereas the dot-dashed line refers to the case of $m_{H}=160 \mathrm{GeV}$. With the full line is reported for comparison the bound coming from BBN and discussed in Eq. (5.4). We can clearly see (shaded region) that for steep enough hypermagnetic energy spectra (i.e., $\epsilon \geq 0.4-0.6$ ) it is possible to have a strongly first-order EW phase transition consistent with the bound (5.4).

a stochastic magnetic field will considerably modify their evolution. In particular, the spherical form of the bubbles is very likely to be spoiled.

Relation (6.2) may be used to define a minimum magnetic field, which can make a phase transition strong enough to allow electroweak baryogenesis in the MSM. One can just fix the Higgs boson mass, find the temperature at which the minimum of the effective potential satisfies the constraint $\varphi_{\min } / T>1$ [36], and read off the hypermagnetic field from Eq. (6.2). With the use of the two-loop effective potential computed in [51], we get, for $m_{H} \sim 80 \mathrm{GeV},\left\langle\left|\overrightarrow{\mathcal{H}}_{Y}\right|^{2}\right\rangle / T_{c}^{4}$ $\geq 0.06$, whereas if $m_{H} \sim 160 \mathrm{GeV}$ we will have $\left\langle\left|\overrightarrow{\mathcal{H}}_{Y}\right|^{2}\right\rangle / T_{c}^{4}$ $\gtrsim 0.3$. For stochastic backgrounds these constraints are plotted in Fig. 8 in terms of our variables $\xi$ and $\epsilon$, characterizing the spectrum. We see that the region of parameter space where a strongly first-order EW phase transition is possible, without spoiling BBN with excessive matter-antimatter domains, extends from $\epsilon \simeq 0.5$ up to $\epsilon>1$. Therefore we come to the conclusion that for violet hypermagnetic energy spectra the level of induced fluctuations is quite tiny at the neutron diffusion distance, but the dynamics of the phase transition can be strongly affected. The magnetic fields, which can modify the nature of the phase transitions, do not appear to be subjected to any other constraints.

The observation that the presence of primordial hypercharge magnetic fields at the electroweak epoch may make an EW phase transition strongly first order removes a main objection against the possibility of baryon asymmetry generation in the MSM [36,49]. We also note that the background magnetic field breaks $C$ and $C P$ symmetries, which may considerably change the analysis of different processes near the domain wall, which are used in EW baryogenesis mechanisms. This study is beyond the scope of this paper. Instead, we will point out in the next subsection that some specific configurations of the hypercharge magnetic field may create the net baryon asymmetry. The following discussion is similar to considerations of the baryogenesis from Chern-Simons condensate in [36].

\section{B. Chern-Simons condensates and the BAU}

In Secs. IV and V we were concerned with the case of stochastically distributed hypermagnetic fields. In this case, the average baryon asymmetry vanishes [i.e., $\left\langle\delta\left(n_{B} /\right.\right.$ $\left.s)\left(\vec{x}, t_{c}\right)\right\rangle=0$ ] in spite of the fact that the fluctuations of the baryon number may be considerable. Thus we assumed that the source of the baryon asymmetry has no relation to the primordial hypermagnetic fields. For example, the BAU may have been generated because of grand unified interactions or at the EW phase transition.

In this subsection we are going to discuss hypermagnetic backgrounds that may give rise to the BAU. We have no idea whether these types of background can or cannot be generated by some mechanism. Our aim is to point out the essential features of the hypermagnetic field that are necessary for the production of net baryon asymmetry.

If the hypermagnetic field configuration is topologically nontrivial (i.e., $\overrightarrow{\mathcal{H}}_{Y} \cdot \vec{\nabla} \times \overrightarrow{\mathcal{H}}_{Y} \neq 0$ ), then $\delta\left(n_{B} / s\right) \neq 0$. As a particular example we will discuss the case of a Chern-Simons wave configuration

$$
\begin{gathered}
\mathcal{Y}_{x}=\mathcal{Y}(t) \sin k_{0} z, \\
\mathcal{Y}_{y}=\mathcal{Y}(t) \cos k_{0} z, \\
\mathcal{Y}_{z}=0 .
\end{gathered}
$$

The hypermagnetic field is $\overrightarrow{\mathcal{H}}=\vec{\nabla} \times \mathcal{Y}$ and the magnetic helicity is simply

$$
\overline{\mathcal{H}} \cdot \bar{\nabla} \times \overline{\mathcal{H}}=k_{0} \mathcal{H}^{2}(t),
$$

with $\mathcal{H}(t)=k_{0} \mathcal{Y}(t)$. Thus from Eq. (6.3) we obtain that

$$
\begin{gathered}
\mathcal{H}_{x}^{2}+\mathcal{H}_{y}^{2}+\mathcal{H}_{z}^{2}=\mathcal{H}^{2}(t), \\
n_{\mathrm{CS}}=-\frac{g^{\prime 2}}{32 \pi^{2}} \overline{\mathcal{H}} \cdot \overline{\mathcal{Y}}=\frac{g^{\prime 2}}{32 \pi^{2} k_{0}} \mathcal{H}^{2}(t) .
\end{gathered}
$$

We notice that this configuration describes a magnetic knot with uniform energy and Chern-Simons density over the whole observable universe. Similar configurations are used in the MHD treatment of the dynamo instability [2]. Inserting the configuration (6.3) into the evolution equations (2.22) and into Eq. (3.1), we get a system of nonlinear ordinary differential equations

$$
\begin{gathered}
\frac{d \mathcal{B}}{d w}=-\frac{4 \alpha^{\prime}}{\pi} \frac{T}{\sigma_{c}}\left(\frac{k_{0}}{T}\right)\left(\frac{\mu_{R}}{T}\right) \mathcal{B}-\frac{T}{\sigma_{c}}\left(\frac{k_{0}}{T}\right)^{2} \mathcal{B}, \quad \mathcal{B}(t)=\frac{\mathcal{H}(t)}{T^{2}} \\
\frac{d}{d w}\left(\frac{\mu_{R}}{T}\right)=-\left(\frac{\alpha^{\prime}}{\pi}\right)^{2} \frac{783}{88}\left(\frac{k_{0}}{\sigma_{c}}\right) \mathcal{B}^{2}-\left(\frac{\Gamma}{T}+\frac{\Gamma_{\mathcal{B}}}{T}\right)\left(\frac{\mu_{R}}{T}\right) \\
\Gamma_{\mathcal{B}}=\frac{\alpha^{\prime}}{\pi} \frac{783}{22} \frac{T}{\sigma_{c}} \mathcal{B}^{2} T
\end{gathered}
$$


(where $w=t T$ ). Equations (6.4) are in fact known as a generalized Lotka-Volterra system [52]. They can be solved numerically for different types of initial conditions. As in the discussion of the stochastic backgrounds, we will consider our system in the adiabatic approximation and we will then use the general set of equations (2.22) and (3.1), valid for arbitrary backgrounds.

As usual, the magnetic diffusivity $\sigma_{c}$ defines the diffusion scale and therefore a Chern-Simons wave configuration with typical momentum $\left(k_{0}\right)$ larger than $k_{\sigma} \sim 10^{-7} T_{c}$ will be washed out. For smaller $k_{0}$, we get from Eq. (3.9)

$$
\begin{aligned}
\left(\frac{n_{B}}{s}\right)\left(\vec{x}, t_{c}\right) & \simeq \frac{\alpha^{\prime}}{2 \pi \sigma_{c}}\left(\frac{n_{f}}{s}\right)\left(\frac{k_{0}}{T_{c}}\right)\left(\frac{M_{0}}{T_{c}}\right) \mathcal{H}^{2}\left(t_{c}\right) \\
& \simeq 10^{10}\left(\frac{k_{0}}{T_{c}}\right)\left(\frac{\mathcal{H}^{2}}{T_{c}^{4}}\right) \text { for } \quad \Gamma \gtrsim \Gamma_{\mathcal{H}}, \\
\left(\frac{n_{B}}{s}\right)\left(\vec{x}, t_{c}\right) & \simeq \frac{11 \pi}{783 \alpha^{\prime}}\left(\frac{n_{f}}{s}\right) k_{0} \Gamma M_{0} \\
& \simeq 0.3\left(\frac{T_{R}}{T_{c}}\right)\left(\frac{k_{0}}{T_{c}}\right) \text { for } \quad \Gamma \lesssim \Gamma_{\mathcal{H}} .
\end{aligned}
$$

Let us now assume that we work only in the framework of the MSM. Then, in order to produce baryon asymmetry we need a strong enough first-order phase transition, and, therefore, a strong enough magnetic field (see preceding subsection). Thus $\Gamma_{\mathcal{H}} \gtrsim \Gamma$. Using the fact that, in the MSM, $T_{R}$ $\simeq 80 \mathrm{TeV}$ we see that $\left(n_{B} / s\right) \simeq 10^{-10}$ for $k_{0} / T_{c} \simeq 10^{-12}$. This value is well below the magnetic diffusivity scale and, therefore, this type of configuration, if ever created, will survive till the EW epoch.

In the extensions of the standard model one may have a strong enough electroweak phase transition without any magnetic field [53]. In addition, the perturbative electron chirality rate may be considerably higher than in the MSM. Thus the hierarchy $\Gamma_{\mathcal{H}} \leqq \Gamma$ may be naturally realized. Then, for $\left(k_{0} / T_{c}\right)\left(\mathcal{H}^{2} / T_{c}^{4}\right) \simeq 10^{-20}$ the BAU calculated from the hypermagnetic field is of the right order of magnitude. For example, if the typical momentum $k_{0}$ of the Chern-Simons condensate is of the order of the EW horizon at $T \sim T_{c}$ (i.e., $k_{0} \sim L_{\mathrm{EW}}^{-1} \simeq 10^{-16} T_{c}$ ) then for small enough hypermagnetic energy (i.e., $\mathcal{H}^{2} \simeq 10^{-4} T_{c}^{4}$ ) the BAU is $\sim 10^{-10}$. Thus it is not excluded that the baryon asymmetry of the universe is a consequence of the topologically nontrivial primordial hypercharge magnetic field.

\section{CONCLUDING REMARKS}

There are no compelling theoretical reasons against the existence of long lived Abelian hypercharge fields at the electroweak epoch. In the present paper we showed that, if they did exist, they could have a number of cosmological consequences. The stochastic hypermagnetic backgrounds induce baryon-number fluctuations because of the electroweak anomaly. These fluctuations may produce sizable matter-antimatter domains at the onset of BBN and affect its predictions. Magnetic fields can change considerably the dynamics of the electroweak phase transition in the MSM and make it strongly first order even for large Higgs masses.
Topologically nontrivial hypermagnetic configurations may be responsible for the BAU.

We left aside a number of questions that may be subjects of future studies. For example, in the study of AMHD equations, we focused our attention on the case where the correlation scale of the velocity field was much smaller than that of the magnetic field, and we also assumed that the velocity field was (globally) invariant under parity transformations (i.e., in the absence of global vorticity over the EW horizon, at the epoch of the phase transition). Owing to different phenomena (e.g., bubble collision) turbulence may arise inside the EW horizon, leading to a non-mirror-symmetric velocity field over some length scale typical of the mechanism responsible for the turbulence. If the turbulence produces a nonzero vorticity of the plasma, then the collective plasma motions may be transformed into fermion number via the amplified hypermagnetic field through a kind of EW dynamo mechanism. We completely neglected the possible occurrence of (global) vorticity, and to relax this hypothesis may be interesting.

We do not know what the possible influence of matterantimatter domains on the inhomogeneous BBN scenario is. In particular, we have no idea if some spectral distribution of hypermagnetic fields could induce a spectrum of baryonnumber fluctuations, which can lead to a larger baryon-tophoton ratio.

\section{ACKNOWLEDGMENTS}

We wish to thank J. Cline, M. Joyce, H. Kurki-Suonio, and G. Veneziano for interesting comments and helpful discussions.

\section{APPENDIX A: MAGNETIC HELICITY CORRELATIONS $\left(\Gamma>\Gamma_{H}\right)$}

The aim of this appendix is to compute explicitly the twopoint correlation function of the magnetic helicity $\Lambda(\vec{x})$ : namely,

$$
\langle\Lambda(\vec{x}) \Lambda(\vec{x}+\vec{r})\rangle=\langle(\vec{H} \cdot \vec{\nabla} \times \vec{H})(\vec{x})(\vec{H} \cdot \vec{\nabla} \times \vec{H})(\vec{x}+\vec{r})\rangle
$$

in terms of the two-point function

$$
G_{i j}(r)=F_{1}(r) \delta_{i j}+r_{i} r_{j} F_{2}(r) .
$$

The results illustrated here are quite relevant for a precise estimate of the level of fluctuations induced by a stochastic background of hypermagnetic fields.

The estimate of the correlation function (A1) may be carried out either in real space or in Fourier space. In Fourier space the calculation can be reduced to the estimate of a convolution, whereas in real space the main algebraic task is to compute the various derivatives appearing in the ensemble average.

The stochastic average appearing in Eq. (A1) can be rewritten as

$$
\langle(\vec{H} \cdot \vec{\nabla} \times \vec{H})(\vec{x})(\vec{H} \cdot \vec{\nabla} \times \vec{H})(\vec{y})\rangle
$$




$$
\begin{aligned}
= & \epsilon_{i j k} \epsilon_{m n l} \lim _{\bar{x}^{\prime} \rightarrow \bar{x}} \lim _{\bar{y}^{\prime} \rightarrow \bar{y}} \frac{\partial}{\partial x^{i}} \frac{\partial}{\partial y^{m}}\left\langle H_{k}\left(\bar{x}^{\prime}\right) H_{j}(\bar{x})\right. \\
& \left.\times H_{l}\left(\bar{y}^{\prime}\right) H_{n}(\bar{y})\right\rangle .
\end{aligned}
$$

This expression turns out to be quite useful, since it allows us to perform the derivations with respect to $x^{i}$ and $y^{m}$ after the average is taken.

If we now use the fact that the background of hypercharge fields is stochastic, we can write that

$$
\begin{aligned}
\left\langle H_{k}\left(\vec{x}^{\prime}\right) H_{j}(\vec{x}) H_{l}\left(\vec{y}^{\prime}\right) H_{n}(\vec{y})\right\rangle & =\left[\left\langle H_{k}\left(\vec{x}^{\prime}\right) H_{j}(\vec{x})\right\rangle\left\langle H_{l}\left(\vec{y}^{\prime}\right) H_{n}(\vec{y})\right\rangle+\left\langle H_{k}\left(\vec{x}^{\prime}\right) H_{l}\left(\vec{y}^{\prime}\right)\right\rangle\right. \\
& \left.\times\left\langle H_{j}(\vec{x}) H_{n}(\vec{y})\right\rangle+\left\langle H_{k}\left(\vec{x}^{\prime}\right) H_{n}(\vec{y})\right\rangle\left\langle H_{j}(\vec{x}) H_{l}\left(\vec{y}^{\prime}\right)\right\rangle\right] .
\end{aligned}
$$

Inserting in Eq. (A4) the representation (A2), we get the following relation:

$$
\begin{aligned}
& \langle(\vec{H} \cdot \vec{\nabla} \times \vec{H})(\vec{x})(\vec{H} \cdot \vec{\nabla} \times \vec{H})(\vec{y})\rangle \\
& =\epsilon_{i j k} \epsilon_{m n l} \lim _{\vec{x}^{\prime} \rightarrow \vec{x} \vec{y}^{\prime} \rightarrow \vec{y}} \lim _{\partial x^{i}} \frac{\partial}{\partial y^{m}}\left[A\left(\vec{x}, \vec{x}^{\prime} ; \vec{y}^{\prime}, \vec{y}^{\prime}\right)\right. \\
& \left.\quad+B\left(\vec{x}, \vec{x}^{\prime} ; \vec{y}, \vec{y}^{\prime}\right)+C\left(\vec{x}, \vec{x}^{\prime} ; \vec{y}^{\prime}, \vec{y}^{\prime}\right)\right],
\end{aligned}
$$

where

$$
\begin{aligned}
A\left(\vec{x}, \vec{x}^{\prime} ; \vec{y}, \vec{y}^{\prime}\right)= & {\left[F_{2}\left(\left|\vec{x}^{\prime}-\vec{x}\right|\right) F_{2}\left(\left|\vec{y}^{\prime}-\vec{y}\right|\right)\left(x_{k}^{\prime}-x_{k}\right)\left(x_{j}^{\prime}-x_{j}\right)\right.} \\
& \times\left(y_{l}^{\prime}-y_{l}\right)\left(y_{n}^{\prime}-y_{n}\right)+F_{1}\left(\left|\vec{x}^{\prime}-\vec{x}\right|\right) F_{2}\left(\mid \vec{y}^{\prime}\right. \\
& -\vec{y} \mid) \delta_{k j}\left(y_{l}^{\prime}-y_{l}\right)\left(y_{n}^{\prime}-y_{n}\right)+F_{2}\left(\mid \vec{x}^{\prime}\right. \\
& -\vec{x} \mid) F_{1}\left(\left|\vec{y}^{\prime}-\vec{y}\right|\right) \delta_{n l}\left(x_{k}^{\prime}-x_{k}\right)\left(x_{j}^{\prime}-x_{j}\right) \\
& \left.+F_{1}\left(\left|\vec{x}^{\prime}-\vec{x}\right|\right) F_{1}\left(\left|\vec{y}^{\prime}-\vec{y}\right|\right) \delta_{k j} \delta_{l n}\right] . \\
B\left(\vec{x}, \vec{x}^{\prime} ; \vec{y}, \vec{y}^{\prime}\right)= & {\left[F_{2}\left(\left|\vec{x}^{\prime}-\vec{y}^{\prime}\right|\right) F_{2}(|\vec{x}-\vec{y}|)\left(x_{k}^{\prime}-y_{k}^{\prime}\right)\left(x_{l}^{\prime}-y_{l}^{\prime}\right)\right.} \\
& \times\left(x_{j}-y_{j}\right)\left(x_{n}-y_{n}\right)+F_{1}\left(\left|\vec{x}^{\prime}-\vec{y}^{\prime}\right|\right) F_{2}(\mid \vec{x} \\
& -\vec{y} \mid) \delta_{k l}\left(x_{j}-y_{j}\right)\left(x_{n}-y_{n}\right)+F_{2}\left(\mid \vec{x}^{\prime}\right. \\
& \left.-\vec{y}^{\prime} \mid\right) F_{1}(|\vec{x}-\vec{y}|) \delta_{n j}\left(x_{k}^{\prime}-y_{k}^{\prime}\right)\left(x_{l}^{\prime}-y_{l}^{\prime}\right) \\
& \left.+F_{1}\left(\left|\vec{x}^{\prime}-\vec{y}^{\prime}\right|\right) F_{1}(|\vec{x}-\vec{y}|) \delta_{k l} \delta_{j n}\right], \\
C\left(\vec{x}, \vec{x}^{\prime} ; \vec{y}, \vec{y}^{\prime}\right)= & {\left[F_{2}\left(\left|\vec{x}^{\prime}-\vec{y}\right|\right) F_{2}\left(\left|\vec{x}-\vec{y}^{\prime}\right|\right)\left(x_{n}^{\prime}-y_{n}^{\prime}\right)\left(x_{k}^{\prime}-y_{k}\right)\right.} \\
& \times\left(x_{j}-y_{j}^{\prime}\right)\left(x_{l}-y_{l}^{\prime}\right)+F_{1}\left(\left|\vec{x}^{\prime}-\vec{y}\right|\right) F_{2}(\mid \vec{x} \\
& \left.-\vec{y}^{\prime} \mid\right) \delta_{k n}\left(x_{j}-y_{j}^{\prime}\right)\left(x_{l}-y_{l}^{\prime}\right)+F_{2}(\mid \vec{x} \\
& \left.-\vec{y}^{\prime} \mid\right) F_{1}\left(\left|\vec{x}^{\prime}-\vec{y}\right|\right) \delta_{l j}\left(x_{k}^{\prime}-y_{k}\right)\left(x_{n}^{\prime}-y_{n}\right) \\
& \left.+F_{1}\left(\left|\vec{x}^{\prime}-\vec{y}\right|\right) F_{1}\left(\left|\vec{x}-\vec{y}^{\prime}\right|\right) \delta_{k n} \delta_{j l}\right] .
\end{aligned}
$$

Recall that, for a generic function $f(r)$ (where $r=|\vec{r}|, r^{a}$ $=x^{a}-y^{a}$ ), the following trivial relation holds:

$\frac{\partial}{\partial x^{i}} \frac{\partial}{\partial y^{m}} f(r)=-\delta_{i m} \frac{1}{r} \frac{\partial f(r)}{\partial r}+\frac{r^{i} r^{m}}{r^{3}} \frac{\partial f(r)}{\partial r}-\frac{r^{i} r^{m}}{r^{2}} \frac{\partial^{2} f(r)}{\partial r^{2}}$.

After having performed the derivatives in Eq. (A5) we can contract the various Levi-Cività tensors with the Kronecker symbols. By then taking the limits indicated in Eqs. (A3) and (A5) we obtain

$$
\begin{aligned}
&\langle(\vec{H} \cdot \vec{\nabla} \times \vec{H})(\vec{x})(\vec{H} \cdot \vec{\nabla} \times \vec{H})(\vec{x}+\vec{r})\rangle \\
&=-\frac{4}{r} F_{1}(r) \frac{d F_{2}(r)}{d r}-2 F_{1}(r) \frac{d^{2} F_{1}(r)}{d r^{2}}+4 r^{2}\left[F_{2}(r)\right]^{2} \\
&+2 r F_{1}(r) \frac{d F_{2}(r)}{d r}-6 r F_{2}(r) \frac{d F_{1}(r)}{d r}+6 F_{1}(r) F_{2}(r) \\
&+2\left(\frac{d F_{1}(r)}{d r}\right)^{2} .
\end{aligned}
$$

In this form the four-point correlation function is completely expressed in terms of the two-point function. Of course we stress that this decomposition holds provided the fields are stochastically distributed, namely, if and only if Eq. (A4) is satisfied.

\section{APPENDIX B: MAGNETIC HELICITY CORRELATIONS $\left(\Gamma<\Gamma_{H}\right)$}

If $\Gamma<\Gamma_{H}$ the correlation function appearing in the final expression of the level of the fluctuations turns out to be the stochastic average of a quantity that contains the magnetic helicity in the numerator and the hypermagnetic energy density in the denominator. Even if this case turns out to be less relevant for the phenomenological considerations presented in the main discussion, we want to outline the main idea that can be used in order to get a large-scale estimate of

$$
\left\langle\left(\frac{\vec{H} \cdot \vec{\nabla} \times \vec{H}}{H^{2}}\right)\left(\vec{x}_{1}\right)\left(\frac{\vec{H} \cdot \vec{\nabla} \times \vec{H}}{H^{2}}\right)\left(\vec{x}_{2}\right)\right\rangle .
$$

The strategy we will use will be to express Eq. (B1) in terms of an appropriate path integral whose functional derivatives will reproduce the correlation function we want to compute. From Eq. (B1) we have, formally

$$
\begin{aligned}
& \left\langle\left(\frac{\vec{H} \cdot \vec{\nabla} \times \vec{H}}{H^{2}}\right)\left(\vec{x}_{1}\right)\left(\frac{\vec{H} \cdot \vec{\nabla} \times \vec{H}}{H^{2}}\right)\left(\vec{x}_{2}\right)\right\rangle \\
& =\lim _{\vec{x}_{1}^{\prime} \rightarrow \vec{x}_{1}} \lim _{\vec{x}_{2}^{\prime} \rightarrow \vec{x}_{2}} \lim _{\alpha \rightarrow 0} \lim _{\beta \rightarrow 0} \epsilon_{i j k} \epsilon_{a b c} \frac{\partial}{\partial x_{1}^{i}} \frac{\partial}{\partial x_{2}^{a}} \\
& \quad \times\left(\frac{\delta}{\delta J_{k}\left(\vec{x}_{1}^{\prime}\right)} \frac{\delta}{\delta J_{j}\left(\vec{x}_{1}\right)} \frac{\delta}{\delta J_{c}\left(\vec{x}_{2}^{\prime}\right)} \frac{\delta}{\delta J_{b}\left(\vec{x}_{2}\right)} \mathcal{W}[\vec{J}]\right)_{\vec{J}=0}
\end{aligned}
$$

where 


$$
\begin{aligned}
\mathcal{W}[\vec{J}]= & \frac{1}{16 \pi^{2}} \int \frac{d^{3} p}{p} \int \frac{d^{3} q}{q} \int D[\vec{H}] \\
& \times \exp \left(-\frac{i}{2} \int d^{3} x \int d^{3} y H_{i}(\vec{x})[K(\vec{x}, \vec{y})]_{i j} H_{j}(\vec{y})\right. \\
& +i \int J_{i}(\vec{x}) H_{i}(\vec{x}) d^{3} x+i p_{i} \int H_{i}(\vec{x}) \delta^{(3)}\left(\vec{x}-\vec{x}_{1}^{\prime}\right) d^{3} x \\
& \left.+i q_{j} \int H_{j}(\vec{x}) \delta^{(3)}\left(\vec{x}-\vec{x}_{2}^{\prime}\right) d^{3} x-\alpha|\vec{p}|^{2}-\beta|\vec{q}|^{2}\right) .
\end{aligned}
$$

In Eq. (B3) we used the fact that formally holds the following relation:

$$
\frac{1}{|\vec{H}|^{2}}=\frac{1}{4 \pi} \lim _{\alpha \rightarrow 0} \int \frac{d^{3} p}{p} e^{i \vec{H} \cdot \vec{p}-\alpha|\vec{p}|^{2}}
$$

By appropriately redefining the source term in the path integral, Eq. (B3) can also be written as

$$
\begin{aligned}
\mathcal{W}[\vec{J}]= & \frac{1}{16 \pi^{2}} \int \frac{d^{3} p}{p} \int \frac{d^{3} q}{q} \int D[\vec{H}] \\
& \times \exp \left\{-\frac{i}{2} \int d^{3} x \int d^{3} y H_{i}(\vec{x})[K(\vec{x}, \vec{y})]_{i j} H_{j}(\vec{y})\right. \\
& \left.-\alpha|\vec{p}|^{2}-\beta|\vec{q}|^{2}+i \int S_{i}(\vec{x}) H_{i}(\vec{x})\right\} d^{3} x
\end{aligned}
$$

where, in the present case,

$$
S_{i}(\vec{x})=J_{i}(\vec{x})+p_{i} \delta^{(3)}\left(\vec{x}-\vec{x}_{1}^{\prime}\right)+q_{i} \delta^{(3)}\left(\vec{x}-\vec{x}_{2}^{\prime}\right) .
$$

By defining

$$
h_{i}(\vec{x})=\int d^{3} y\left[K^{1 / 2}(\vec{x}, \vec{y})\right]_{i j} H_{j}(\vec{y})
$$

we obtain that the argument of the exponential can be expressed as

$$
\begin{gathered}
\frac{i}{2} \int d^{3} x\left\{h_{i}(\vec{x})-\int d^{3} x S_{i}(\vec{y})\left[K^{-1 / 2}(\vec{x}, \vec{y})\right]_{i j}\right\}^{2} \\
-\frac{i}{2} \int d^{3} x \int d^{3} y S_{i}(\vec{x}) G_{i j}(|\vec{x}-\vec{y}|) S_{j}(\vec{y}) .
\end{gathered}
$$

Notice that the symbol $[K(\vec{x}, \vec{y})]_{i j}$ must have the following properties, which will be important also for the calculation of the functional integral:

$$
\begin{gathered}
\int d^{3} y\left[K^{1 / 2}(\vec{x}, \vec{y})\right]_{i j}\left[K^{1 / 2}(\vec{y}, \vec{z})\right]_{j k}=[K(\vec{x}, \vec{z})]_{i k}, \\
\int d^{3} y\left[K^{1 / 2}(\vec{x}, \vec{y})\right]_{i j}\left[K^{-1 / 2}(\vec{y}, \vec{z})\right]_{j k}=\delta_{i k} \delta^{(3)}(\vec{x}-\vec{z}), \\
\int d^{3} y\left[K^{-1 / 2}(\vec{x}, \vec{y})\right]_{i j}\left[K^{-1 / 2}(\vec{y}, \vec{z})\right]_{j k}=\left[K(\vec{x}, \vec{z})^{-1}\right]_{i k},
\end{gathered}
$$

$$
\left[K^{-1}(\vec{x}, \vec{y})\right]_{i j}=-G_{i j}(|\vec{x}-\vec{y}|) .
$$

We can integrate the part that is quadratic in the fields; then $\mathcal{W}[\bar{J}]$ becomes

$$
\begin{aligned}
\mathcal{W}[\bar{J}]= & W[0] \int \frac{d^{3} p}{p} \int \frac{d^{3} q}{q} \\
& \times \exp \left(-\frac{i}{2} \int d^{3} x \int d^{3} y S_{i}(\bar{x}) G_{i j}(|\bar{x}-\bar{y}|) S_{j}(\bar{y})\right. \\
& \left.-\alpha|\bar{p}|^{2}-\beta|\bar{q}|^{2}\right)
\end{aligned}
$$

where $\mathcal{W}[0]$ is the usual Jacobian. Using the definition of $S_{i}(\vec{x})$ we obtain for $\mathcal{W}[\bar{J}]$ :

$$
\begin{aligned}
\frac{\mathcal{W}[\vec{J}]}{\mathcal{W}[0]}= & \int \frac{d^{3} p}{p} \int \frac{d^{3} q}{q} e^{C\left(q_{i}, p_{i} ; q_{j}, p_{j}\right)} \\
& \times \exp \left\{-\frac{1}{2} \int d^{3} x \int d^{3} y J_{i}(\vec{x}) G_{i j}(|\vec{x}-\vec{y}|) J_{j}(\vec{y})\right. \\
& \left.-i \int d^{3} x J_{l}(x) L_{l}(x)\right\},
\end{aligned}
$$

with

$$
L_{l}(x)=p_{m} G_{m l}\left(\left|\vec{x}-\vec{x}_{1}^{\prime}\right|\right)+q_{m} G_{m l}\left(\left|\vec{x}-\vec{x}_{2}^{\prime}\right|\right)
$$

and

$$
\begin{aligned}
C\left(q_{i}, p_{i} ; q_{j}, p_{j}\right)= & -\frac{1}{2} q_{i} G_{i j}(0) q_{j}-\frac{1}{2} p_{i} G_{i j}(0) p_{j} \\
& -q_{i} G_{i j}\left(\left|\vec{x}_{1}^{\prime}-\vec{x}_{2}^{\prime}\right|\right) p_{j}-\alpha|\vec{p}|^{2}-\beta|\vec{q}|^{2}
\end{aligned}
$$

$\left[G_{i j}(0)\right.$ comes because there are two $\delta$ functions centered at the same point for the terms quadratic in $q_{i}$ and $p_{i}$ ]. Performing the functional derivatives we obtain (evaluating the generating function for $J=0$ )

$$
\begin{aligned}
\left(\frac{\delta}{\delta J_{k}\left(\vec{x}_{1}^{\prime}\right)} \frac{\delta}{\delta J_{j}\left(\vec{x}_{1}\right)} \frac{\delta}{\delta J_{c}\left(\vec{x}_{2}^{\prime}\right)} \frac{\delta}{\delta J_{b}\left(\vec{x}_{2}\right)} W[\vec{J}]\right)_{\vec{J}=0} \\
=\left\{\left[G_{k j}\left(\left|\vec{x}_{1}-\vec{x}_{1}^{\prime}\right|\right) G_{c b}\left(\left|\vec{x}_{2}-\vec{x}_{2}^{\prime}\right|\right)+G_{k c}\left(\left|\vec{x}_{1}^{\prime}-\vec{x}_{2}^{\prime}\right|\right)\right.\right. \\
\left.\quad \times G_{j b}\left(\left|\vec{x}_{1}-\vec{x}_{2}\right|\right)+G_{k b}\left(\left|\vec{x}_{1}^{\prime}-\vec{x}_{2}\right|\right) G_{j c}\left(\left|\vec{x}_{1}-\vec{x}_{2}^{\prime}\right|\right)\right] \\
\quad-L_{b}\left(\vec{x}_{2}\right) L_{c}\left(\vec{x}_{2}^{\prime}\right) G_{j k}\left(\left|\vec{x}_{1}-\vec{x}_{1}^{\prime}\right|\right)-L_{k}\left(\vec{x}_{1}^{\prime}\right) L_{j}\left(\vec{x}_{1}\right) \\
\quad \times G_{b c}\left(\left|\vec{x}_{2}-\vec{x}_{2}^{\prime}\right|\right)-L_{c}\left(\vec{x}_{2}^{\prime}\right) L_{k}\left(\vec{x}_{1}^{\prime}\right) G_{b j}\left(\left|\vec{x}_{1}-\vec{x}_{2}\right|\right) \\
\quad-L_{b}\left(\vec{x}_{2}\right) L_{k}\left(\vec{x}_{1}^{\prime}\right) G_{c j}\left(\left|\vec{x}_{1}-\vec{x}_{2}^{\prime}\right|\right)-L_{c}\left(\vec{x}_{2}^{\prime}\right) L_{j}\left(\vec{x}_{1}\right) \\
\quad \times G_{b k}\left(\left|\vec{x}_{1}^{\prime}-\vec{x}_{2}\right|\right)-L_{b}\left(\vec{x}_{2}\right) L_{k}\left(\vec{x}_{1}^{\prime}\right) G_{k c}\left(\left|\vec{x}_{1}^{\prime}-\vec{x}_{2}^{\prime}\right|\right) \\
\left.\quad+L_{k}\left(\vec{x}_{1}^{\prime}\right) L_{j}\left(\vec{x}_{1}\right) L_{b}\left(\vec{x}_{2}^{\prime}\right) L_{c}\left(\vec{x}_{2}\right)\right\} \mathcal{W}[0] .
\end{aligned}
$$

Notice that the fifth and sixth terms of Eq. (B12) vanish when contracted with the $\epsilon$ tensors. In order to perform the 
integration over $p$ and $q$, we have to expand the expression giving us the correlation function for

$$
g(r)=\frac{\mathcal{G}(R)}{\mathcal{G}(0)}<1,
$$

[see also Eq. (4.11)]. This approximation holds for sufficiently large scales, provided the Green functions decay for $R \gg 1$. This requirement is automatically satisfied in our case, since we only consider the situation where the energy spectrum is increasing in frequency (i.e., blue or violet spectra). We now take the limits for $\vec{x}_{1}^{\prime} \rightarrow \vec{x}_{1}$, and for $\vec{x}_{2}^{\prime} \rightarrow \vec{x}_{2}$ and Eq. (B2) becomes

$$
\begin{aligned}
& \left\langle\left(\frac{\vec{H} \cdot \vec{\nabla} \times \vec{H}}{H^{2}}\right)(\vec{x})\left(\frac{\vec{H} \cdot \vec{\nabla} \times \vec{H}}{H^{2}}\right)(\vec{x}+\vec{r})\right\rangle \\
& \simeq \lim _{\alpha \rightarrow 0} \lim _{\beta \rightarrow 0} \frac{1}{16 \pi^{2}} \int \frac{d^{3} p}{p} \int \frac{d^{3} q}{q}\left[A_{1}(r)+q^{2} A_{2}(r)\right. \\
& \left.\quad-p^{2} A_{3}(r)+2 A_{4}(r)(\vec{q} \cdot \vec{p})\right] \exp \left(-\frac{\left\langle H^{2}(\vec{x})\right\rangle}{2}\left(q^{2}+p^{2}\right)\right. \\
& \left.\quad-\alpha|\vec{q}|^{2}-\beta|\vec{p}|^{2}+O(g(r))\right),
\end{aligned}
$$

where

$$
\begin{gathered}
A_{1}(r)=\langle(\vec{H} \cdot \vec{\nabla} \times \vec{H})(\vec{x})(\vec{H} \cdot \vec{\nabla} \times \vec{H})(\vec{x}+\vec{r})\rangle, \\
A_{2}(r)=A_{3}(r)=\frac{1}{2}\left[\left\langle H^{2}(\vec{x})\right\rangle\right]^{3} g(r),
\end{gathered}
$$

$$
A_{4}(r)=\frac{1}{16}\left[\left\langle H^{2}(\vec{x})\right\rangle\right]^{3} g(r) \text {. }
$$

We can now integrate over $q$ and $p$. We notice that since the integral is convergent also for $\alpha, \beta \rightarrow 0$ the limits can be taken before the integration. It is convenient to perform the integration over $q$ and $p$ separately; in this way, after angular integration, the apparently Gaussian integrals can be expressed as ordinary exponential integrals of the type

$$
\int_{0}^{\infty} d \lambda \lambda^{n} e^{-a \lambda}=\frac{\Gamma(n+1)}{a^{n+1}} .
$$

After integration, $A_{2}(r)$ and $A_{3}(r)$ cancel whereas the contribution of the term containing $A_{4}(r)$ vanishes because of the angular integration. The final result obtained in the assumption that the Green functions decay at large distances is then

$$
\begin{aligned}
& \left\langle\left(\frac{\vec{H} \cdot \vec{\nabla} \times \vec{H}}{H^{2}}\right)(\vec{x})\left(\frac{\vec{H} \cdot \vec{\nabla} \times \vec{H}}{H^{2}}\right)(\vec{x}+\vec{r})\right\rangle \\
& \quad \simeq \frac{\langle(\vec{H} \cdot \vec{\nabla} \times \vec{H})(\vec{x})(\vec{H} \cdot \vec{\nabla} \times \vec{H})(\vec{x}+\vec{r})\rangle}{\left\langle H^{2}(\vec{x})\right\rangle^{2}}+O(g(r)),
\end{aligned}
$$

which is exactly what we report in Sec. IV. The method used in the present appendix can also be exploited in order to compute further corrections, if needed.
[1] J. Schwinger, Phys. Rev. 75, 1912 (1949).

[2] Y. B. Zeldovich, A. A. Rusmaikin, and D. D. Sokoloff, Magnetic Fields in Astrophysics (Gordon and Breach, New York, 1983); E. N. Parker, Cosmical Magnetic Fields (Clarendon, Oxford, 1979).

[3] P. P. Kronberg, Rep. Prog. Phys. 57, 325 (1994).

[4] S. I. Vainshtein and Ya. B. Zeldovich, Usp. Fiz. Nauk. 106, 431 (1972) [Sov. Phys. Usp. 15, 159 (1972)]; W. H. Matthaeus, M. L. Goldstein, and S. R. Lantz, Phys. Fluids 29, 1504 (1986).

[5] R. M. Kulsrud and S. W. Anderson, Astrophys. J. 396, 606 (1992).

[6] E. R. Harrison, Nature (London) 224, 1090 (1969); Mon. Not. R. Astron. Soc. 147, 279 (1970); Phys. Rev. Lett. 30, 188 (1973).

[7] J. Quashnock, A. Loeb, and D. N. Spergel, Astrophys. J. 344, L49 (1989).

[8] B. Cheng and A. V. Olinto, Phys. Rev. D 50, 2421 (1994); G. Sigl, A. V. Olinto, and K. Jedamzik, ibid. 55, 4582 (1997).

[9] T. W. Kibble and A. Vilenkin, Phys. Rev. D 52, 679 (1995).

[10] T. Vachaspati, Phys. Lett. B 265, 258 (1991).

[11] K. Enqvist and P. Olesen, Phys. Lett. B 319, 178 (1993); 329, 195 (1994); P. Olesen, ibid. 398, 321 (1997).

[12] G. Baym, D. Bodeker, and L. McLerran, Phys. Rev. D 53, 662 (1996)
[13] M. Joyce and M. Shaposhnikov, Phys. Rev. Lett. 79, 1193 (1997).

[14] L. P. Grishchuk, Zh. Eksp. Teor. Fiz. 67, 825 (1974) [Sov. Phys. JETP 40, 409 (1975)]; Usp. Fiz. Nauk 156, 297 (1988) [Sov. Phys. Usp. 31, 940 (1988)].

[15] L. Parker, Phys. Rev. Lett. 21, 562 (1968); Phys. Rev. 183, 1057 (1969).

[16] M. S. Turner and L. M. Widrow, Phys. Rev. D 37, 2743 (1988).

[17] B. Ratra, Astrophys. J. Lett. 391, L1 (1992).

[18] M. Gasperini, M. Giovannini, and G. Veneziano, Phys. Rev. Lett. 75, 3796 (1995); D. Lemoine and M. Lemoine, Phys. Rev. D 52, 1955 (1995).

[19] M. Gasperini, M. Giovannini, and G. Veneziano, Phys. Rev. D 52, 6651 (1995).

[20] G. Greenstein, Nature (London) 223, 938 (1969); J. J. Matese and R. F. O 'Connel, Astrophys. J. 160, 451 (1970); B. Cheng, D. N. Schramm, and J. Truran, Phys. Rev. D 49, 5006 (1994); B. Cheng, A. Olinto, D. N. Schramm, and J. Truran, ibid. 54, 4714 (1996); D. Grasso and H. Rubinstein, Astropart. Phys. 3, 95 (1995); Phys. Lett. B 379, 73 (1996); P. Kernan, G. Starkman, and T. Vachaspati, Phys. Rev. D 54, 7207 (1996).

[21] J. Barrow, P. Ferreira, and J. Silk, Phys. Rev. Lett. 78, 3610 (1997). 
[22] J. Barrow, Phys. Rev. D 55, 7451 (1997).

[23] A. Kosowsky and A. Loeb, Astrophys. J. 461, 1 (1996); M. Giovannini, Phys. Rev. D 56, 3198 (1997).

[24] T. Kolatt, "Determination of the Primordial Magnetic Field Power Spectrum by Faraday Rotation Correlations,' Report No. astro-ph/9704243.

[25] N. A. Krall and A. W. Trivelpiece, Principles of Plasma Physics (San Francisco Press, San Francisco, 1986).

[26] M. Giovannini and M. E. Shaposhnikov, Phys. Rev. Lett. 80, 22 (1998)

[27] K. Kajantie, M. Laine, K. Rummukainen, and M. Shaposhnikov, Nucl. Phys. B493, 413 (1997).

[28] V. Rubakov and A. Tavkhelidze, Phys. Lett. 165B, 109 (1985); V. Rubakov, Prog. Theor. Phys. 75, 366 (1986).

[29] C. P. Dettmann, N. E. Frankel, and V. Kowalenko, Phys. Rev. D 48, 5655 (1993); R. M. Gailis, C. P. Dettmann, N. E. Frankel, and V. Kowalenko, ibid. 50, 3847 (1994); A. Sil, N. Banerjee, and S. Chatterjee, ibid. 53, 7369 (1996).

[30] D. Biskamp, Non-linear Magnetohydrodynamics (Cambridge University Press, Cambridge, England, 1994).

[31] B. Campbell, S. Davidson, J. Ellis, and K. Olive, Phys. Lett. B 297, 118 (1992); L. E. Ibanez and F. Quevedo, ibid. 283, 261 (1992); J. M. Cline, K. Kainulainen, and K. A. Olive, Phys. Rev. Lett. 71, 2372 (1993); Phys. Rev. D 49, 6394 (1994).

[32] A. N. Redlich and L. C. R. Wijewardhana, Phys. Rev. Lett. 54, 970 (1985).

[33] M. Joyce, T. Prokopec, and N. Turok, Phys. Rev. D 53, 2930 (1996); G. Baym and H. Heiselberg, ibid. 56, 5254 (1997).

[34] S. Yu. Khlebnikov and M. E. Shaposhnikov, Phys. Lett. B 387, 817 (1996).

[35] V. A. Kuzmin, V. A. Rubakov, and M. E. Shaposhnikov, Phys. Lett. 155B, 36 (1985).

[36] M. E. Shaposhnikov, Pis'ma Zh. Eksp. Teor. Fiz. 44, 364 (1986) [JETP Lett. 44, 465 (1986)]; Nucl. Phys. B287, 757 (1987); B299, 797 (1988).

[37] I. S. Gradshteyn and I. M. Ryzhik, Tables of Integrals, Series and Products, 5th ed. (Academic Press, New York, 1994), p. 529.

[38] Handbook of Mathematical Functions, edited by M. Abramowitz and I. A. Stegun (Dover, New York, 1972).

[39] R. A. Malaney and G. J. Mathews, Phys. Rep. 229, 145 (1993); S. Sarkar, Rep. Prog. Phys. 59, 1493 (1996).

[40] E. Lifshitz and L. P. Pitaevskii, Physical Kinetics (Pergamon Press, Oxford, 1981).

[41] K. Jedamzik and G. M. Fuller, Astrophys. J. 423, 33 (1994).

[42] K. Jedamzik, G. M. Fuller, and G. J. Mathews, Astrophys. J. 43, 50 (1994).

[43] H. Kurki-Suonio, K. Jedamzik, and G. J. Mathews, Astrophys. J. 479, 31 (1997).

[44] J. Applegate, C. J. Hogan, and R. J. Scherrer, Phys. Rev. D 35, 1151 (1987).

[45] K. Jedamzik and G. M. Fuller, Astrophys. J. 452, 33 (1995).

[46] R. Brandenberger, A. C. Davis, and M. J. Rees, Phys. Lett. B 349, 329 (1995).

[47] A. Dolgov and J. Silk, Phys. Rev. D 47, 4244 (1993).

[48] K. Jedamzik, V. Katalinic, and A. Olinto, "Damping of Cosmic Magnetic Fields," Report No. astro-ph/9606080.

[49] V. A. Rubakov and M. E. Shaposhnikov, Usp. Fiz. Nauk 166, 493 (1996) [Phys. Usp. 39, 461 (1996)]; A. G. Cohen, D. B. Kaplan, and A. E. Nelson, Annu. Rev. Nucl. Part. Sci. 43, 27 (1993); A. D. Dolgov, Phys. Rep. 222, 309 (1992).

[50] E. Lifshitz and L. P. Pitaevskii, Statistical Physics, Part 2 (Pergamon Press, Oxford, 1981).

[51] K. Kajantie, M. Laine, K. Rummukainen, and M. E. Shaposhnikov, Nucl. Phys. B458, 90 (1996); B466, 189 (1996).

[52] R. Grimshaw, Non-linear Ordinary Differential Equations (Blackwell Scientific Publications, Oxford, 1990).

[53] M. Carena, M. Quiros, and C. E. M. Wagner, Phys. Lett. B 380, 81 (1996); M. Laine, Nucl. Phys. B481, 43 (1996); J. M. Cline and K. Kainulainen, ibid. B482, 73 (1996); M. Losada, Phys. Rev. D 56, 2893 (1997). 